(c) 2006 International Press

Adv. Theor. Math. Phys. 10 (2006) 77-121

\title{
GLSMs for Gerbes (and other toric stacks)
}

\author{
${ }^{1}$ Department of Mathematics, University of Pennsylvania, \\ David Rittenhouse Laboratory, 209 South 33rd Street, Philadelphia, \\ PA 19104-6395, USA \\ ${ }^{2}$ Departments of Physics and Mathematics, University of Utah, \\ Salt Lake City, UT 84112, USA \\ tpantev@math.upenn.edu,ersharpe@math.utah.edu
}

\begin{abstract}
In this paper, we will discuss gauged linear sigma model descriptions of toric stacks. Toric stacks have a simple description in terms of (symplectic, GIT) $\mathbf{C}^{\times}$quotients of homogeneous coordinates, in exactly the same form as toric varieties. We describe the physics of the gauged linear sigma models that formally coincide with the mathematical description of toric stacks and check that physical predictions of those gauged linear sigma models exactly match the corresponding stacks. We also see in examples that when a given toric stack has multiple presentations in a form accessible as a gauged linear sigma model, that the IR physics of those different presentations matches, so that the IR physics is presentation-independent, making it reasonable to associate CFTs to stacks, not just presentations of stacks. We discuss mirror symmetry for stacks, using MorrisonPlesser-Hori-Vafa techniques to compute mirrors explicitly, and also find a natural generalization of Batyrev's mirror conjecture. In the process of studying mirror symmetry, we find some new abstract CFTs, involving fields valued in roots of unity.
\end{abstract}

e-print archive: http://lanl.arXiv.org/abs/hep-th/0502053 


\section{CONTEnts}

$\begin{array}{ll}\text { 1. Introduction } & 79\end{array}$

2. Two-dimensional gauge theories with nonminimal charges 81

3. Gerbes over projective spaces and quantum cohomology 83

3.1. First example: $\mathbf{Z}_{k}$ gerbe on $\mathbf{P}^{N-1} \quad 83$

3.2. Alternate presentation of the first example 89

3.3. More gerbes on projective spaces and alternative $\begin{array}{ll}\text { presentations } & 92\end{array}$

4. More general toric stacks 94

4.1. Stacks over Hirzebruch surfaces 95

4.2. Analogue of flops 95

$\begin{array}{ll}\text { 4.3. Weighted projective stacks } & 96\end{array}$

4.4. Quantum cohomology for toric stacks 97

5. Calabi-Yau gerbes 98

5.1. Example: three-fold in $G_{-1}^{k} \mathbf{P}^{4}$ and Landau-Ginzburg $\begin{array}{ll}\text { orbifolds } & 99\end{array}$

5.2. Example: Calabi-Yau in $G_{-n}^{k} \mathbf{P}^{N-1}$ and GLSM phases 100

6. Mirrors to stacks 102

6.1. Toda theories corresponding to gerbes on projective spaces 102

6.1.1. Toda theory for $G_{-1}^{k} \mathbf{P}^{N-1} \quad 102$

6.1.2. Toda theory for $G_{-n}^{k} \mathbf{P}^{N-1} \quad 103$

6.1.3. Check of correlation functions of Toda duals 104

6.2. Mirrors to gerbes on the quintic 108 


\section{Introduction}

String compactifications on stacks are described in [1]. Briefly, every ${ }^{1}$ stack has a presentation as a global quotient by a group $[X / G]$. A sigma model on such a presentation is simply a $G$-gauged sigma model on $X$. Now, a given stack can have many presentations of that form, yielding very different physical theories. However, we have argued in [1], and will check further here, that the gauged sigma models defined by different presentations of a single stack all lie in the same universality class of worldsheet RG flow. This we take to be the central consistency condition for the notion of "string compactification on a stack" to be well-defined. In checking consistency of this hypothesis, one runs into many potential obstacles, perhaps most importantly a mismatch between physical deformations and stack deformations; these issues are addressed in detail in [1].

Just as toric varieties provide a nontrivial set of examples that are easy to analyse, there is a notion of toric stacks [2], which have analogous properties. Just as toric varieties can be described by gauged linear sigma models, so too can toric stacks, and this paper is devoted to explaining the relevant physics of such gauged linear sigma models.

A gauged linear sigma model describing a toric stack looks very much like a gauged linear sigma model describing a toric variety, except that the charges with respect to some of the $U(1)$ 's will typically be nonminimal. Now, due to peculiar properties of two-dimensional quantum field theories, a two-dimensional gauge theory with fields of nonminimal charges is not the

\footnotetext{
${ }^{1}$ Under extremely mild technical conditions, essentially irrelevant for physics, as explained in [1].
} 
same as a two-dimensional gauge theory with fields of minimal charges the two physical theories are distinguished by nonperturbative effects, as we shall explicitly see shortly.

Just as the physics of the two-dimensional $\mathbf{C P}^{N-1}$ model plays an important role in understanding the physics of traditional gauged linear sigma models, the physics of analogous models describing gerbes over projective spaces, which we shall call the $G \mathbf{P}^{N-1}$ models, play an important role in understanding gauged linear sigma models for toric stacks, and we shall spend much of this paper describing such models. A gerbe, the reader should recall, is a local orbifold by a noneffectively acting group, and as with all local orbifolds, can be expressed as a global quotient by a larger group. The $G \mathbf{P}^{N-1}$ models we shall consider correspond to $\mathbf{Z}_{k}$ gerbes over projective spaces, meaning that they describe local orbifolds by trivially acting $\mathbf{Z}_{k}$ 's. The easiest nontrivial example of such a gerbe can be expressed in a form almost identical to the $\mathbf{C P}^{N-1}$ model, except that the fields all have charge $k$ rather than charge 1 , which is one way to describe a local orbifold by a noneffectively acting $\mathbf{Z}_{k}$.

We begin in Section 2 by giving a general explanation for why twodimensional gauge theories with nonminimal charges are physically distinct from two-dimensional gauge theories with minimal charges. The basic reason is nonperturbative effects: the charge dictates which line bundle a Higgs field couples to, and different line bundles mean different zero modes, hence different anomalies, different correlation functions, and thus different physical theories. We shall see many examples of this distinction throughout this paper.

In Section 3, we study examples of $\mathbf{Z}_{k}$ gerbes over projective spaces, which we describe using gauged linear sigma models (in which the fields have nonminimal $U(1)$ charges). We compute physical properties of these models, such as quantum cohomology rings. Since we can compute quantum cohomology rings without knowing classical cohomology rings, our computations give a very nontrivial check of the massless spectrum conjecture presented in [1]. We also are able to check presentation-independence of universality classes, as some of these gerbes have multiple realizations in gauged linear sigma models of rather different-looking forms, so, e.g., we can repeat calculations in each different gauged linear sigma model and check that the results are independent of presentation.

In Section 4, we briefly outline some more general examples of toric stacks and their properties. We briefly outline the analogue of flops for stacks. We also briefly discuss weighted projective stacks - just like weighted projective spaces, except singularities are replaced by local orbifold structures. 
We observe that the quantum cohomology results of [3] immediately generalize to toric stacks.

In Section 5, we discuss Calabi-Yau gerbes, realized as complete intersections in toric stacks. Physically, the corresponding gauged linear sigma models are easy to describe: for each $U(1)$, the degree of the hypersurface must match the sum of the charges of the chiral superfields, just as usual. This turns out to give an easy physics derivation of a mathematical result that all gerbes over Calabi-Yau's are themselves Calabi-Yau. We study phases of the analogue of the quintic in a gerbe over $\mathbf{P}^{4}$ and use the spectrum of the Landau-Ginzburg orbifold (now a noneffective orbifold, an extension of the usual $\mathbf{Z}_{5}$ by a trivially acting $\mathbf{Z}_{k}$ ) to give a very strong check of the general massless spectrum conjecture presented in [1] for strings on stacks.

In Section 6, we study mirror symmetry for stacks. We begin by computing Toda duals to the gerbes over projective spaces described in Section 3. In addition to getting another check of presentation-independence, by verifying that the Toda dual depends upon the gerbe and not the presentation thereof, we find that the Toda duals contain fields valued in roots of unity, a result we verify by checking correlation functions in the Toda duals and comparing to quantum cohomology results from Section 3. Such fields are (to the authors) rather unusual in physics, though we also saw them from independent lines of reasoning in [4], when studying marginal deformations of noneffective orbifolds. We discuss the mirror of the gerby quintic, and gerby minimal models, and present a conjecture for a generalization of Batyrev's mirror conjecture to hypersurfaces in toric stacks. Now, the data defining a toric stack looks much like the data defining a toric variety, except that the fan is decorated by some abelian finite-group data. By adding fields valued in roots of unity to terms in superpotentials of Landau-Ginzburg models, for example, we generate Newton polyhedra with symmetric data, making an analogue of Batyrev's conjecture possible. Note that, yet again, we see fields valued in roots of unity.

Finally, in an appendix, we review the [2] description of toric stacks.

\section{Two-dimensional gauge theories with nonminimal charges}

Since the gauged linear sigma models for many toric stacks describe chiral superfields with nonminimal $U(1)$ charges, let us take a few moments to explain in general terms why nonperturbative effects distinguish such theories from two-dimensional gauge theories with minimal charges. Later we shall see numerous physical properties that differ between the theories, 
from correlation functions to R-symmetry anomalies, but in this section, we shall just review the basic principles. (We would like to thank J. Distler and R. Plesser for providing the detailed argument that we give in this section.) For a different discussion of two-dimensional gauge theories with fermions of nonminimal charges, see [5, Section 4]. (The discussion there is most applicable to the present situation when $m \ll M$, in the notation of that reference.)

To be specific, consider a gauged linear sigma model with a single $U(1)$ gauge field, and with chiral superfields, all of charge $k$, with $k>1$. (Mathematically, this corresponds to a gerbe on a projective space, as we shall review in the next section.) One might argue that this theory should be the same as a theory with chiral superfields of charge 1, as follows. Since instanton number is essentially monopole number, from Dirac quantization since the electrons have charges a multiple of $k$, the instantons must have charge a multiple of $1 / k$, and so zero modes of the Higgs fields in a minimal nonzero instanton background would be sections of $\mathcal{O}(k / k)=\mathcal{O}(1)$, just as in a minimal charge GLSM. Making the charges nonminimal has not changed the physics. In order to recover the physics we have described, we require the Higgs fields to have charge $k$ while the instanton numbers are integral, not fractional.

Closer analysis reveals subtleties. Let us break up the analysis into two separate cases: first, the case that the worldsheet is noncompact and second, that the worldsheet is compact. For both cases, it will be important that the worldsheet theory is two-dimensional.

First, the noncompact case. Since the $\theta$ angle couples to $\operatorname{Tr} F$, we can determine the instanton numbers through the periodicity of $\theta$. Suppose we have the physical theory described above, namely a GLSM with Higgs fields of charge $k$, plus two more massive fields, of charges +1 and -1 . In a two-dimensional theory, the $\theta$ angle acts as an electric field, which can be screened by pair production, and that screening determines the periodicity of $\theta$. If the only objects we could pair produce were the Higgs fields of charge $k$, then the theta angle would have periodicity $2 \pi k$, and so the instanton numbers would be multiples of $1 / k$. However, since the space is noncompact, and the electric field fills the entire space, we can also pair produce arbitrary numbers of the massive fields, which have charges \pm 1 , and so the $\theta$ angle has periodicity $2 \pi$, so the instantons have integral charges.

We can phrase this more simply as follows. In a theory with only Higgs fields of charge $k$, the instanton numbers are multiples of $1 / k$, and so the resulting physics is equivalent to that of a GLSM with minimal charges. However, if we add other fields of charge \pm 1 , then the instanton numbers are integral, and if those fields become massive, and we work at an energy 
scale below that of the masses of the fields, then we have a theory with Higgs fields of charge $k$, and integral instanton numbers, giving us the physics that corresponds to a gerbe target.

Thus, we see in the noncompact case that there are two possible physical theories described by Higgs fields of charge $k$ : one is equivalent to the GLSM with minimal charges, and the other describes the gerbe.

The analysis for the compact worldsheet case is much shorter. Strictly speaking, to define the theory nonperturbatively on a compact space, we must specify, by hand, the bundles that the Higgs fields couple to. If the gauge field is described by a line bundle $L$, then coupling all of the Higgs fields to $L^{\otimes k}$ is a different prescription from coupling all of the Higgs fields to $L$. As a result, the spectrum of zero modes differs between the two theories, hence correlation functions and anomalies differ between the two theories, and so the two physical theories are very different, as we shall see in examples later.

We shall assume throughout this paper that the worldsheet is compact, though as we have argued the same subtlety shows up for noncompact worldsheets.

We are interested in these physical theories because they often crop up in describing stacks. Since stacks are defined via their incoming maps, a precise definition of which bundles the superfields couple to is part of the definition of the stack, and so there is no ambiguity in which physical theory to associate to a given (presentation of a) stack.

\section{Gerbes over projective spaces and quantum cohomology}

In this section, we will discuss basic properties of physical theories describing gerbes over projective spaces, the gerby analogue of the old "CP${ }^{N-1}$ models." We will occasionally refer to these gerby analogues as $G \mathbf{P}^{N-1}$ models, or more specifically, $G_{a}^{k} \mathbf{P}^{N-1}$ models, for a $\mathbf{Z}_{k}$ gerbe on $\mathbf{P}^{N-1}$ classified by

$$
a \bmod k \in \mathbf{Z}_{k}=H^{2}\left(\mathbf{P}^{N-1}, \mathbf{Z}_{k}\right)
$$

In this notation, therefore, $G_{a}^{k} \mathbf{P}^{N-1}=G_{a+k}^{k} \mathbf{P}^{N-1}$.

\subsection{First example: $\mathbf{Z}_{k}$ gerbe on $\mathbf{P}^{N-1}$}

Let us begin with an easy example of a gerbe over projective space $\mathbf{P}^{N-1}$. The projective space itself is described by a gauged linear sigma model with 
$N$ chiral superfields $x_{i}$, each of charge one with respect to a gauged $U(1)$. Mathematically, the projective space is the quotient

$$
\mathbf{P}^{N-1}=\frac{\mathbf{C}^{N}-0}{\mathbf{C}^{\times}}
$$

We can describe gerbes on projective spaces in a very similar fashion. In particular, the $\mathbf{Z}_{k}$ gerbe on $\mathbf{P}^{N-1}$ that generates all of the $\mathbf{Z}_{k}$ gerbes is given by the quotient

$$
\left[\frac{\mathbf{C}^{N}-0}{\mathbf{C}^{\times}}\right]
$$

where the $\mathbf{C}^{\times}$acts by $k$ times the minimal amount. The characteristic class of this gerbe is

$$
-1 \in \mathbf{Z}_{k}=H^{2}\left(\mathbf{P}^{N-1}, \mathbf{Z}_{k}\right)
$$

essentially because $\mathbf{C}^{N}-0$ with the natural projection to $\mathbf{P}^{N-1}$ is the total space of $\mathcal{O}_{\mathbf{P}^{N-1}}(-1)$. Clearly, this corresponds to a gauged linear sigma model with $N$ chiral superfields $x_{i}$, each of charge $k$ under a gauged $U(1)$.

So, in this example, one describes a gerbe over a projective space, instead of a projective space, simply by multiplying all the charges by $k$.

We obtained the description of nonminimal charges from the description of the quotient, but there is also another way to obtain this description, and that is directly from the definition of the gerbe. Described as a quotient stack $\left[\left(\mathbf{C}^{N}-0\right) / \mathbf{C}^{\times}\right]$, a map into the gerbe is a pair consisting of a principal $\mathbf{C}^{\times}$bundle $L$ over the worldsheet, together with a $\mathbf{C}^{\times}$-equivariant map from the total space of $L$ into $\mathbf{C}^{N}-0$, where the $\mathbf{C}^{\times}$acts by $k$ times the usual amount. Now, mathematically, a pair consisting of a principal $\mathbf{C}^{\times}$bundle $P$ together with a map $P \rightarrow \mathbf{C}$, equivariant with respect to a $\mathbf{C}^{\times}$action that rotates the $\mathbf{C} k$ times, is equivalent to a pair consisting of a line bundle $L$ together with a section of $L^{\otimes k}$. We can see this as follows. Starting with the pair $(P, \phi: \operatorname{tot}(P) \rightarrow \mathbf{C})$, construct the pair $\left(L, s \in H^{0}\left(X, L^{k}\right)\right)$ by setting $L=P \times_{\chi} \mathbf{C}$ and $s(x)=[(p, \phi(p))]$. Here we have:

- $\chi: \mathbf{C}^{\times} \rightarrow \mathbf{C}^{\times}$is the character $\alpha \rightarrow \alpha^{k}$;

- $P \times{ }_{\chi} \mathbf{C}$ is the associated line bundle. It is given explicitly as the quotient of $P \times \mathbf{C}$ by the $\mathbf{C}^{\times}$action in which $\alpha \in \mathbf{C}^{\times}$acts on $(p, z)$ as $\left(p \cdot \alpha^{-1}, \alpha^{-k} z\right)$.

- $[(p, \phi(p))] \in L_{x}$ denotes the $\mathbf{C}^{\times}$orbit of the point $(p, \phi(p)) \in P_{x} \times \mathbf{C}$. Note that $[(p, \phi(p))]$ is independent of the choice of $p \in P_{x}$ since $\phi$ is $\chi$-equivariant. 
Conversely, given $(L, s)$, take $P$ to be the frame bundle of $L$, i.e., $L$ with the zero section removed. The pullback of $L$ to $P$ is canonically trivialized since the tautological section is a frame. The pullback of $s$ to $P$ is then a section of the trivial bundle, i.e., a map from $P$ to $\mathbf{C}$. It is then straightforward to check that this map is equivariant. In particular, a set of not-simultaneously vanishing maps from $\operatorname{tot}(P)$ to $\mathbf{C}$ is equivalent to a set of not-simultaneously-vanishing Higgs fields. Thus, the data describing the gerbe as a quotient stack is equivalent to data describing line bundles with sections of $L^{\otimes k}$ (taking into account D-terms), which is manifestly the description of the Higgs fields. Thus, one can recover the nonminimal charge description directly from the definition of the gerbe.

Before describing how the nonperturbative physics of this two-dimensional gauge theory differs from that of the ordinary $\mathbf{C P}^{N-1}$ model, let us take a moment to understand why this two-dimensional gauge theory should correspond to the gerbe. In the ordinary $\mathbf{C P}^{N-1}$ model, D-terms restrict the classical Higgs vacua to a sphere $S^{2 N-1}$, so gauging $U(1)$ rotations leaves

$$
\frac{S^{2 N-1}}{U(1)}=\mathbf{P}^{N-1}
$$

In the present case, we are gauging a $U(1)$ that acts by $k$ rotations of the $U(1)$ fibres of the principal $U(1)$ bundle over $\mathbf{P}^{N-1}$ whose total space is the sphere $S^{2 N-1}$. Since the $U(1)$ rotates $k$ times instead of once, locally this is the same as a trivially acting $\mathbf{Z}_{k}$ orbifold of $\mathbf{P}^{N-1}$ - the expectation values of the Higgs fields do not completely break the gauge symmetry, but only break it to a noneffectively acting subgroup. However, because the sphere $S^{2 N-1}$ is a nontrivial $U(1)$ bundle, the resulting local orbifold is not the same as the global orbifold $\left[\mathbf{P}^{N-1} / \mathbf{Z}_{k}\right]$.

We have seen in numerous examples in [4] that orbifolding by a noneffectively acting group does not reproduce the original theory. To be consistent, one would expect that two-dimensional gauge theories with nonminimal charges must differ from two-dimensional gauge theories with minimal charges.

Perturbatively in QFT, multiplying all the charges by a nonzero constant has absolutely no effect on the theory. However, nonperturbatively, it can have an effect, as the charges determine which bundles each field couples to, as discussed in Section 2. For example, in a degree $d$ instanton background, in the gauged linear sigma model describing merely $\mathbf{P}^{N-1}$, the $x_{i}$ are holomorphic sections of $\mathcal{O}(d)$, whereas in the linear sigma model describing the gerbe, the $x_{i}$ are holomorphic sections of $\mathcal{O}(k d)$. 
Just as in the ordinary $\mathbf{C P}^{N-1}$ model, where the axial $U(1)_{R}$ is broken to $\mathbf{Z}_{2 N}$ by anomalies, here the same $U(1)_{R}$ is broken to $\mathbf{Z}_{2 k N}$. We can derive this from e.g., [6, equation (3.4)], or alternatively, since the lowenergy theory can be approximated by the $\mathbf{C P}^{N-1}$ model with a restriction that degrees of maps are divided by $k$ (as we shall see explicitly in a few moments), we can do a Riemann-Roch computation in the worldsheet A model. Furthermore, following the methods of [5], it is natural to conjecture that the IR limit of this theory develops a mass gap and has $k N$ vacua, just as the ordinary $\mathbf{C P}^{N-1}$ model has a gap and $N$ vacua. We shall confirm that speculation in Section 6.1, when we derive and analyse the Toda theory corresponding to this model.

Now that we have begun to see some of the reasons why this theory is physically distinct from the ordinary $\mathbf{C} \mathbf{P}^{N-1}$ model, let us check the relation between this theory and the mathematics of gerbes.

The first check we shall perform involves the Witten index. In the ordinary $\mathbf{C P}^{N-1}$ model, the Witten index $\operatorname{Tr}(-)^{F}$ computes the Euler characteristic of the target, $\mathbf{P}^{N-1}$. In the present case, given a mass gap and $k N$ vacua in the IR, the Witten index of the present theory is $k N$. Now, as described in more detail in [1], the relevant notion of Euler characteristic for stacks is the Euler characteristic of the associated inertia stack (the "orbifold Euler characteristic," for stacks presented as global quotients by finite groups), and for the gerbe at hand, the Euler characteristic of the associated inertia stack is precisely $k N$, in agreement with the Witten index.

Now, let us study linear sigma model moduli spaces and quantum cohomology and compare these physical properties to what one expects mathematically for the gerbe.

The linear sigma model moduli space is obtained by expanding worldsheet fields in a basis of zero modes. The coefficients in the expansion are the homogeneous coordinates on the moduli space and are given the same $U(1)$ charges as the original field. In the present case, for what morally are degree $d$ maps, we have that $x_{i} \in \Gamma(\mathcal{O}(k d))$ for the gauged linear sigma model with nonminimal charges. We exclude coefficients such that all the $x_{i} \equiv 0$, leaving us with

$$
\mathcal{M}=\left[\frac{\mathbf{C}^{N(k d+1)}-0}{\mathbf{C}^{\times}}\right]
$$

where all fields have charge $k$ with respect to the $\mathbf{C}^{\times}$.

If the fields all had charge 1 with respect to the $\mathbf{C}^{\times}$, then $\mathcal{M}=\mathbf{P}^{N(k d+1)-1}$. However, since they have charge $k$, if we are careful about the quotient, 
then we must interpret the moduli "space" $\mathcal{M}$ as itself being a stack, and in particular, a $\mathbf{Z}_{k}$ gerbe over $\mathbf{P}^{N(k d+1)-1}$.

Before computing quantum cohomology, let us compare these physical statements to the mathematics of maps into gerbes. First, each map from $\mathbf{P}^{1}$ into a $\mathbf{Z}_{k}$ gerbe has a $\mathbf{Z}_{k}$ 's worth of automorphisms. If we keep track of those automorphisms, then our moduli "space" of maps does not have a set of points, so much as a category of points, in which each point is replaced by a copy of the classifying stack $B \mathbf{Z}_{k}$. This is exactly the local structure of a $\mathbf{Z}_{k}$ gerbe. In other words, on mathematical grounds, because maps from the worldsheet into the gerbe have automorphisms, we should expect that the moduli 'space' should have the structure of a gerbe, a $\mathbf{Z}_{k}$ gerbe in fact, and that is exactly what we see in the linear sigma model moduli space.

We can check even more. Our nonperturbative interpretation of nonminimal charges ensures that the degree of each map in the moduli space is a multiple of $k$. This physical consequence of nonminimal charges has a mathematical explanation in terms of gerbes. Since there is a projection map from the $\mathbf{Z}_{k}$ gerbe into the underlying manifold $\mathbf{P}^{N-1}$, each map from the worldsheet $\mathbf{P}^{1}$ into the gerbe also defines a map $f$ from $\mathbf{P}^{1}$ into $\mathbf{P}^{N-1}$. Now, a map from $\mathbf{P}^{1}$ into a gerbe $\mathcal{G}$ is the same as a map $f$ into $\mathbf{P}^{N-1}$ together with a trivialization ${ }^{2}$ of $f^{*} \mathcal{G}$. Equivalently, the natural map

$$
f^{*}: H^{2}\left(\mathbf{P}^{N-1}, \mathbf{Z}_{k}\right) \longrightarrow H^{2}\left(\mathbf{P}^{1}, \mathbf{Z}_{k}\right)
$$

should be identically zero, otherwise the pullback $f^{*} \mathcal{G}$ would not have a trivialization. But

$$
H^{2}\left(\mathbf{P}^{N-1}, \mathbf{Z}_{k}\right)=H^{2}\left(\mathbf{P}^{1}, \mathbf{Z}_{k}\right)=\mathbf{Z}_{k}
$$

and the natural map $f^{*}: \mathbf{Z}_{k} \rightarrow \mathbf{Z}_{k}$ is multiplication by the degree of the map. Hence, for a gerbe on $\mathbf{P}^{N-1}$ of characteristic class $n \bmod k$, if we let $d$

\footnotetext{
${ }^{2} \mathrm{~A}$ map from $\mathbf{P}^{1}$ into the gerbe $\mathcal{G}$ over $M$ defines a map from $\mathbf{P}^{1}$ into the fibre product $\mathbf{P}^{1} \times{ }_{M} \mathcal{G}=f^{*} \mathcal{G}$, and such a map is a trivialization. This is seen perhaps most easily if we replace gerbes with bundles. If $\mathcal{G}$ is a bundle over a space $X$, and we are given a map $f: \mathbf{P}^{1} \rightarrow X$, then since there is a canonical map $f^{*} \mathcal{G} \rightarrow \mathcal{G}$, a map $\mathbf{P}^{1} \rightarrow f^{*} \mathcal{G}$ can be composed with that canonical map to give a map $g: \mathbf{P}^{1} \rightarrow \mathcal{G}$, such that $f=\pi \circ g$ for $\pi: \mathcal{G} \rightarrow X$. Conversely, given a map $g: \mathbf{P}^{1} \rightarrow \mathcal{G}$, if we let $f=\pi \circ g$, then we can define a map $\mathbf{P}^{1} \rightarrow f^{*} \mathcal{G}$ as follows. Recall

$$
f^{*} \mathcal{G}=\left\{(x, e) \in \mathbf{P}^{1} \times \mathcal{G} \mid f(x)=\pi(e)\right\}
$$

so given $g: \mathbf{P}^{1} \rightarrow \mathcal{G}$, we can map $x \mapsto(x, g(x)) \in f^{*} \mathcal{G}$, for $x \in \mathbf{P}^{1}$. The argument for $\mathcal{G}$ a gerbe instead of a bundle is virtually identical.
} 
denote the degree of the map $f: \mathbf{P}^{1} \rightarrow \mathbf{P}^{N-1}$, then we have that

$$
d(n \bmod k)=0 \bmod k
$$

In the present case, $n=-1$, so we have that $d$ must be divisible by $k$. Conversely, any map from $\mathbf{P}^{1} \rightarrow \mathbf{P}^{N-1}$ of degree a multiple of $k$, together with a section of the trivial gerbe on $\mathbf{P}^{1}$, defines a map from the worldsheet $\mathbf{P}^{1}$ into the gerbe.

Thus, not only does the fact that the linear sigma model moduli "space" is a gerbe has a mathematical interpretation in terms of properties of maps into gerbes, but also the fact that the maps have degree a multiple of $k$ also naturally agrees with what one expects for gerbes. Put more simply, the linear sigma model moduli 'space' is a compactification of the moduli space of maps into the gerbe.

Next let us outline a physical prediction for the quantum cohomology of this gerbe, which will also further illustrate how the physical theory for the gerbe is distinct from the physical theory for the underlying space.

For the projective space $\mathbf{P}^{N-1}$, the linear sigma model moduli space for degree $d$ maps is given by $\mathbf{P}^{N(d+1)-1}$, so the A model has correlation functions of the form $\left\langle X^{N(d+1)-1}\right\rangle=q^{d}$ (with $X$ corresponding to the generator of degree two cohomology) defining ${ }^{3}$ a quantum cohomology relation $X^{N}=q$.

For the gerbe under consideration, we have seen that the linear sigma model moduli space is given by a $\mathbf{Z}_{k}$ gerbe over $\mathbf{P}^{N(k d+1)-1}$, so from the dimension of the moduli space, we see that in the gerbe theory, we have correlators of the form $\left\langle X^{N(k d+1)-1}\right\rangle=q^{d}$ (with $X$ a pullback to the gerbe of degree two cohomology), defining a quantum cohomology relation $X^{k N}=q$. Since the quantum cohomology ring of this gauged linear sigma model with nonminimal charges is not isomorphic to the quantum cohomology ring of the underlying space $\mathbf{P}^{N-1}$, or more simply, the correlation functions are different, we see that the physical theory corresponding to the gerbe really is different from the theory with minimal charges.

There is another way to derive this quantum cohomology relation in the gerbe theory, using methods of [3]. By using one-loop effective actions, the authors of [3] derived a general expression for quantum cohomology for toric

\footnotetext{
${ }^{3}$ One fast way to think about the quantum cohomology relations in simple cases is that they can be used to generate all of the correlators from the classical correlation functions. Here, if $X$ generates degree two cohomology, then since $\mathbf{P}^{N-1}$ is $(N-1)$-dimensional, the nonzero classical correlation function is $\left\langle X^{N-1}\right\rangle=1$, so we can use the relation $X^{N}=q$ to derive the general correlation function above from the classical correlation function.
} 
targets from the gauged linear sigma model. Applying [3, equation (3.44)] to the present example, we find

$$
\prod_{1}^{N}(k \sigma)^{k}=\exp (2 \pi i \tau)
$$

or, after appropriate redefinitions, we have that

$$
\sigma^{k N}=q
$$

the same quantum cohomology relation we derived above.

\subsection{Alternate presentation of the first example}

In this section, let us discuss an alternate presentation of the gerbe discussed in the previous section, as a check that the physics captured mathematically by the stack is presentation-independent.

Consider a gauged linear sigma model with $N$ chiral superfields $x_{i}$, and another chiral superfield $z$, with two gauged $U(1)$ 's, under which the chiral superfields have charges

\begin{tabular}{c|cc} 
& $x_{i}$ & $z$ \\
\hline$\lambda$ & 1 & -1 \\
$\mu$ & 0 & $k$
\end{tabular}

Associated to each $U(1)$ is a D-term:

$$
\begin{aligned}
\sum_{i}\left|x_{i}\right|^{2}-|z|^{2} & =r_{\lambda} \\
k|z|^{2} & =r_{\mu}
\end{aligned}
$$

We shall assume that $r_{\mu} \gg 0$, so that $z \neq 0$. In this case, were we not gauging the second $U(1)$, the resulting toric variety would be the total space of the principal $U(1)$ bundle over $\mathbf{P}^{N-1}$ of degree -1 . By gauging the second $U(1)$, we are gauging rotations of the fibre of that principal $U(1)$ bundle. If $k=1$, the resulting toric variety is, mathematically, $\mathbf{P}^{N-1}$. If $k>1$, then mathematically we have a $\mathbf{Z}_{k}$ gerbe on $\mathbf{P}^{N-1}$, the same gerbe as that discussed in the last section.

Let us study the physics of this gauged linear sigma model and check that if $k>1$, not only is the nonperturbative physics distinct from that of the $\mathbf{P}^{N-1}$ model, but also that the A model twist of this gauged linear sigma model has isomorphic properties to the model discussed in the last section (so that the physics is presentation-independent, essentially). 
In fact, before doing detailed calculations, we can immediately perform a quick test ${ }^{4}$ that this really is an alternate presentation of the first example. Note that by redefining $\mathbf{C}^{\times}$actions, we can map this model to one described by homogeneous coordinates with weights as follows:

$$
\begin{array}{c|cc} 
& x_{i} & z \\
\hline \lambda^{k} \mu & k & 0 \\
\mu & 0 & k
\end{array}
$$

This is almost the same as the model we started with, except for the extra homogeneous coordinate. In fact, this model describes the previous presentation of the gerbe, tensored with a single homogeneous coordinate modded out by a $\mathbf{C}^{\times}$that acts with weight $k$ - which is a presentation of the trivial gerbe $B \mathbf{Z}_{k}=\left[\right.$ point $/ \mathbf{Z}_{k}$. Now, our map between $\mathbf{C}^{\times}$actions has nontrivial kernel; schematically, we have a short exact sequence

$$
0 \longrightarrow \mathbf{Z}_{k} \longrightarrow(\lambda, \mu) \longrightarrow\left(\lambda^{k} \mu, \mu\right) \longrightarrow 0
$$

so, we can relate the theories defined by the two sets of $\mathbf{C}^{\times}$actions as

$$
\left(\lambda^{k} \mu, \mu\right)=\left[\frac{(\lambda, \mu)}{\mathbf{Z}_{k}}\right]
$$

The $\mathbf{Z}_{k}$ quotient on the right side of the equation above acts trivially, globally, and as discussed in [1], the corresponding physical theory of such a global trivial quotient is a tensor product

$$
\left(\lambda^{k} \mu, \mu\right)=(\lambda, \mu) \otimes\left[\frac{\text { point }}{\mathbf{Z}_{k}}\right]
$$

In particular, note this is precisely consistent with our underlying conjecture that stacks classify universality classes of gauged sigma models. The $\left(\lambda^{k} \mu, \mu\right)$ theory is, explicitly, a tensor product of the theory defined by the first presentation of the $\mathbf{Z}_{k}$ gerbe over $\mathbf{P}^{N-1}$, with a theory defined by a presentation of the trivial gerbe $B \mathbf{Z}_{k}=\left[\right.$ point $\left./ \mathbf{Z}_{k}\right]$. Our analysis of $\mathbf{C}^{\times}$ actions reveals that the $\left(\lambda^{k} \mu, \mu\right)$ theory is also a tensor product of the theory defined by the $(\lambda, \mu)$ presentation of the $\mathbf{Z}_{k}$ gerbe over $\mathbf{P}^{N-1}$ with a presentation of the trivial gerbe $B \mathbf{Z}_{k}$. These two descriptions of the $\left(\lambda^{k}, \mu\right)$ theory have matching universality classes, so long as stacks really do classify universality classes.

Now that we have analysed $\mathbf{C}^{\times}$actions, let us turn to other tests of our conjecture that stacks classify universality classes of gauged sigma models.

\footnotetext{
${ }^{4}$ We would like to thank K. Hori for suggesting this test to us.
} 
The linear sigma model moduli space is now defined by two integers $d_{1}$, $d_{2}$, associated to the gauged $U(1)$ 's $\lambda, \mu$, respectively. The zero modes of the chiral superfields are given by

$$
\begin{aligned}
x_{i} & \in \Gamma\left(\mathcal{O}\left(d_{1}\right)\right) \\
z & \in \Gamma\left(\mathcal{O}\left(-d_{1}+k d_{2}\right)\right)
\end{aligned}
$$

Let us assume for simplicity that $d_{1} \geq 0$ and $-d_{1}+k d_{2} \geq 0$. Then, following the same analysis as before, the linear sigma model moduli "space" looks like a $\mathbf{Z}_{k}$ gerbe over $\mathbf{P}^{N\left(d_{1}+1\right)-1} \times \mathbf{P}^{-d_{1}+k d_{2}}$, where the homogeneous coordinates $x_{i a}$ and $z_{b}$ (from the zero modes of the $x_{i}$ and $z$ ) have weights under two $U(1)$ 's as

\begin{tabular}{c|cc} 
& $x_{i a}$ & $z_{b}$ \\
\hline$\lambda$ & 1 & -1 \\
$\mu$ & 0 & $k$
\end{tabular}

From the dimensions of the moduli spaces, correlators in an A model twist of a lower energy theory look like they should have two generators, $X, Y$, with correlation functions

$$
\left\langle X^{N\left(d_{1}+1\right)-1} Y^{-d_{1}+k d_{2}}\right\rangle=q_{1}^{d_{1}} q_{2}^{d_{2}}
$$

which given the classical correlation function $\left\langle X^{N-1}\right\rangle=1$ define quantum cohomology relations

$$
\begin{aligned}
X^{N} Y^{-1} & =q_{1} \\
Y^{k} & =q_{2}
\end{aligned}
$$

Note, however, that the quantum cohomology ring with two generators $X$, $Y$ and relations as above is isomorphic to the ring with one generator $X$ and relation $X^{k N}=q$ (for $q=q_{2} q_{1}^{k}$ ). In other words, the quantum cohomology ring of this theory is isomorphic to the quantum cohomology ring of the theory of Section 3.1. This means that the A model correlators are isomorphic between these two theories, exactly as one would expect if they lie in the same universality class.

Let us check this result by using the methods of [3]. Applying [3, equation (3.44)] to the present case, we have

$$
\begin{aligned}
\left(\prod_{1}^{N} \sigma_{1}\right)\left(-\sigma_{1}+k \sigma_{2}\right)^{-1} & =\exp \left(2 \pi i \tau_{1}\right) \\
\left(-\sigma_{1}+k \sigma_{2}\right)^{k} & =\exp \left(2 \pi i \tau_{2}\right)
\end{aligned}
$$


By making the identifications

$$
\begin{aligned}
X & \sim \sigma_{1} \\
Y & \sim-\sigma_{1}+k \sigma_{2}
\end{aligned}
$$

we recover the quantum cohomology relations derived previously.

\subsection{More gerbes on projective spaces and alternative presentations}

Let us begin by describing a $\mathbf{Z}_{k}$ gerbe on $\mathbf{P}^{N-1}$ with characteristic class in $H^{2}\left(S^{2}, \mathbf{Z}_{k}\right)$ given by $-n \bmod k$, i.e., the $G_{-n}^{k} \mathbf{P}^{N-1}$ model.

Mathematically, we can describe this gerbe as a $\mathbf{C}^{\times}$quotient of the total space of a principal $\mathbf{C}^{\times}$bundle over $\mathbf{P}^{N-1}$, with the property that $c_{1} \bmod k=$ $-n \bmod k$, in which the $\mathbf{C}^{\times}$rotates the fibres $n$ times.

To describe that quotient, consider a linear sigma model with $N$ chiral superfields $x_{i}$ plus $z$, and two $\mathbf{C}^{\times}$actions, with weights as shown:

\begin{tabular}{c|cc} 
& $x_{i}$ & $z$ \\
\hline$\lambda$ & 1 & $-n$ \\
$\mu$ & 0 & $k$
\end{tabular}

We shall assume that $n$ is positive. The D-terms are

$$
\begin{array}{r}
\sum_{i}\left|x_{i}\right|^{2}-n|z|^{2}=r_{\lambda} \\
k|z|^{2}=r_{\mu}
\end{array}
$$

We shall assume that $r_{\mu} \gg 0$ and $r_{\lambda} \gg 0$. If $r_{\mu} \neq 0$, then $z \neq 0$, and the remaining D-term disallows $x_{i}=0$ for all $i$. Thus, we are quotienting

$$
\left(\mathbf{C}^{N}-0\right) \times \mathbf{C}^{\times}
$$

by a pair of $\mathbf{C}^{\times}$actions.

Mathematically, this gauged linear sigma model should describe a gerbe over $\mathbf{P}^{N-1}$ classified by $-n \bmod k$, and we shall check that physical properties of this gauged linear sigma model are reflected in mathematical properties of the gerbe.

Since shifting $n \mapsto n+k$ changes the presentation but leaves the gerbe invariant, the statement that stacks classify universality classes implies that the pertinent physics should be invariant under $n \mapsto n+k$, and we shall check that statement. 
Let us consider curve counting in this gerbe, following the same procedure as in the last few examples. First, note that a single integer no longer suffices to specify the degree of the maps, we must specify two integers $d_{1}, d_{2}$. Then, expanding fields in zero modes to get the linear sigma model moduli space, we have

$$
\begin{aligned}
x_{i} & \in H^{0}\left(\mathbf{P}^{1}, \mathcal{O}\left(d_{1}\right)\right)=\mathbf{C}^{d_{1}+1} \\
z & \in H^{0}\left(\mathbf{P}^{1}, \mathcal{O}\left(-n d_{1}+k d_{2}\right)\right)=\mathbf{C}^{-n d_{1}+k d_{2}+1}
\end{aligned}
$$

(For simplicity, we shall assume that $d_{1} \geq 0$ and $-k d_{1}+n d_{2} \geq 0$.) The resulting moduli space $\mathcal{M}$ can be described in GLSM language as $N\left(d_{1}+1\right)$ homogeneous coordinates $x_{i a},-n d_{1}+k d_{2}+1$ homogeneous coordinates $z_{b}$, with a pair of $\mathbf{C}^{\times}$actions under which the homogeneous coordinates have weights

\begin{tabular}{c|cc} 
& & \\
& $x_{i a}$ & $z_{b}$ \\
\hline$\lambda^{\prime}$ & 1 & $-n$ \\
$\mu^{\prime}$ & 0 & $k$
\end{tabular}

The resulting linear sigma model moduli "space" is a $\mathbf{Z}_{k}$-gerbe over a projectivization of the vector bundle $\mathcal{O}(-n)^{\oplus-n d_{1}+k d_{2}+1}$ over $\mathbf{P}^{N\left(d_{1}+1\right)-1}$, or, more simply, a $\mathbf{Z}_{k}$-gerbe over $\mathbf{P}^{-n d_{1}+k d_{2}} \times \mathbf{P}^{N\left(d_{1}+1\right)-1}$.

As before, given the moduli space, in a simple example of this form, we can now compute quantum cohomology in the A model twist of a lower energy theory. Correlators have two generators $X, Y$, and from the dimension of the moduli space, we can read off the correlation functions

$$
\left\langle X^{N\left(d_{1}+1\right)-1} Y^{-n d_{1}+k d_{2}}\right\rangle=q_{1}^{d_{1}} q_{2}^{d_{2}}
$$

From these correlation functions, it is clear that with respect to the generators $X, Y$, the quantum cohomology ring is defined by the relations

$$
\begin{aligned}
X^{N} Y^{-n} & =q_{1} \\
Y^{k} & =q_{2}
\end{aligned}
$$

Note that we immediately recover the previous example as a special case.

Note that if we shift $n \mapsto n+k$, which leaves the gerbe invariant, the resulting moduli space is equivalent to the moduli space with $d_{1}^{\prime}=d_{1}$ and $d_{2}^{\prime}=-d_{1}+d_{2}$. Since physically we sum over all degrees, A model physics can only depend upon $n \bmod k$. Similarly, under $n \mapsto n+k$, the quantum cohomology ring is invariant - the new relations are equivalent to the old ones, with $q_{1}^{\prime}=q_{1} / q_{2}$. Thus, A model results only depend upon $n$ and $k$ in the combination $n \bmod k$, consistent with the assumption that universality 
classes of gauged sigma models are classified by stacks, not by presentations thereof.

Let us check this quantum cohomology calculation by using the methods of [3]. Applying [3, equationn (3.44)] to the present case gives us

$$
\begin{aligned}
\left(\prod_{1}^{N} \sigma_{1}\right)\left(-n \sigma_{1}+k \sigma_{2}\right)^{-n} & =\exp \left(2 \pi i t_{1}\right) \\
\left(-n \sigma_{1}+k \sigma_{2}\right)^{k} & =\exp \left(2 \pi i t_{2}\right)
\end{aligned}
$$

If we make the identifications

$$
\begin{aligned}
& X \sim \sigma_{1} \\
& Y \sim-n \sigma_{1}+k \sigma_{2}
\end{aligned}
$$

then we recover the previous expression for quantum cohomology.

The reader should note that the quantum cohomology relations we have derived give a product structure on the cohomology of the inertia stacks associated to these gerbes, which all look like $k$ disjoint copies of the gerbe. In computing quantum cohomology, starting only with what should be untwisted sector fields, we have implicitly recovered twist fields as well. Also note this provides a check of the claim in [1] that the massless spectrum of a string on a stack should be counted by cohomology of the inertia stack.

In passing, note that when $n=0$, i.e., when the gerbe is trivial, the quantum cohomology ring above is a product of the quantum cohomology of the ordinary $\mathbf{P}^{N-1}$ model and a twist field, agreeing with general results on factorizability of physical theories associated to trivial gerbes presented in [4].

\section{More general toric stacks}

For the most part, we concentrate in this paper on the special case of gauged linear sigma models for $\mathbf{Z}_{k}$ gerbes over projective spaces, as in our opinion those give very clear illustrations of the pertinent physics. Gauged linear sigma models for more general toric stacks are very similar, in that they typically look like gauged linear sigma models on toric varieties, except that the charges with respect to some of the $U(1)$ 's are nonminimal. The only exceptions are those toric stacks whose GLSM description is identical to that traditionally associated to certain special toric varieties, as we shall discuss in Subsection 4.3. 


\subsection{Stacks over Hirzebruch surfaces}

Just to give some easy examples of more general toric stacks and their corresponding gauged linear sigma models, let us consider Hirzebruch surfaces. Recall that these surfaces, traditionally denoted $\mathbf{F}_{n}$ and indexed by integers, correspond to total spaces of $\mathbf{P}^{1}$-bundles over $\mathbf{P}^{1}$ 's. They can be described with gauged linear sigma models very simply as follows. Let $s, t, u, v$ be homogeneous coordinates; then the gauged linear sigma model has two $U(1)$ 's, call them $\lambda, \mu$, under which the homogeneous coordinates have charges as follows:

\begin{tabular}{c|cccc} 
& $s$ & $t$ & $u$ & $v$ \\
\hline$\lambda$ & 1 & 1 & $n$ & 0 \\
$\mu$ & 0 & 0 & 1 & 1
\end{tabular}

The homogeneous coordinates $s, t$ act as homogeneous coordinates on the base of the $\mathbf{P}^{1}$ bundle, and the coordinates $u, v$ act as homogeneous coordinates on the fibre of the $\mathbf{P}^{1}$ bundle.

Suppose you wanted to describe a $\mathbf{Z}_{k}$ gerbe over the base of the $\mathbf{P}^{1}$ bundle. Then, a gauged linear sigma model for such a toric stack would have homogeneous coordinates $s, t, u, v$, with charges

\begin{tabular}{c|cccc} 
& $s$ & $t$ & $u$ & $v$ \\
\hline$\lambda$ & $k$ & $k$ & $k n$ & 0 \\
$\mu$ & 0 & 0 & 1 & 1
\end{tabular}

Similarly, a $\mathbf{Z}_{k}$ gerbe over the fibre of the $\mathbf{P}^{1}$ bundle could be described as

\begin{tabular}{c|cccc} 
& $s$ & $t$ & $u$ & $v$ \\
\hline$\lambda$ & 1 & 1 & $n$ & 0 \\
$\mu$ & 0 & 0 & $k$ & $k$
\end{tabular}

and so forth.

\subsection{Analogue of flops}

In gauged linear sigma models studied in the past, varying the FayetIliopoulos parameters through different geometric phases has the effect of realizing birational transformations. The same statement is true of gauged linear sigma models describing toric stacks, for the basic reason that varying the Fayet-Iliopoulos parameters only changes the exceptional set of 
the quotient, so on a Zariski-open subset, the resulting stacks will be isomorphic.

Let us illustrate this with a gerby analogue of a classic example from [7] - flopping between the two small resolutions of a conifold singularity.

Consider the linear sigma model with five chiral superfields $a, b, c, d, e$, and $\mathbf{C}^{\times}$actions as below:

\begin{tabular}{c|ccccc} 
& $a$ & $b$ & $c$ & $d$ & $e$ \\
\hline$\lambda$ & 1 & 1 & -1 & -1 & $k$ \\
$\mu$ & 0 & 0 & 0 & 0 & $n$
\end{tabular}

If we omitted $e$ and $\mu$, then we would recover the small resolutions of the affine conifold. The corresponding $\mathrm{D}$ terms are

$$
\begin{aligned}
|a|^{2}+|b|^{2}-|c|^{2}-|d|^{2}+k|e|^{2} & =r_{\lambda} \\
n|e|^{2} & =r_{\mu}
\end{aligned}
$$

We shall assume that $r_{\mu} \gg 0$, so that $e \neq 0$. Integrating out $e$ leaves us with a single D-term given by

$$
|a|^{2}+|b|^{2}-|c|^{2}-|d|^{2}=r_{\lambda}-\left(\frac{k}{n}\right) r_{\mu}
$$

When $r_{\lambda}-(k / n) r_{\mu} \ll 0$, the D-term dictates an exceptional set $\{c=d=0\}$, so that we are left with the quotient

$$
\frac{\mathbf{C}^{2} \times\left(\mathbf{C}^{2}-0\right) \times \mathbf{C}^{\times}}{\mathbf{C}^{\times} \times \mathbf{C}^{\times}}
$$

which appears to be a $\mathbf{Z}_{n}$ gerbe over one small resolution of the conifold.

When $r_{\lambda}-(k / n) r_{\mu} \gg 0$, the D-term gives the exceptional set $\{a=b=0\}$. We are left with the quotient

$$
\frac{\left(\mathbf{C}^{2}-0\right) \times \mathbf{C}^{2} \times \mathbf{C}^{\times}}{\mathbf{C}^{\times} \times \mathbf{C}^{\times}}
$$

describing a gerbe over the other small resolution.

\subsection{Weighted projective stacks}

We have so far discussed toric stacks that can be described by gauged linear sigma models with nonminimal charges. However, there are some toric 
stacks whose corresponding gauged linear sigma models look identical to that traditionally associated to the underlying space.

The easiest examples of such toric stacks are the weighted projective stacks. These are defined in exactly the same way as weighted projective spaces, except that one takes a stacky quotient instead of an ordinary quotient. For example, a weighted projective space $W \mathbf{P}_{k_{1}, k_{2}, \ldots}^{n}$ is defined as the quotient

$$
\frac{\mathbf{C}^{n+1}-\{0\}}{\mathbf{C}^{\times}}
$$

where the $\mathbf{C}^{\times}$acts on the homogeneous coordinates with weights $\left(k_{1}, k_{2}, \ldots\right)$. The corresponding weighted projective stack is defined in almost exactly the same way as the stack quotient

$$
\left[\frac{\mathbf{C}^{n+1}-\{0\}}{\mathbf{C}^{\times}}\right]
$$

where the $\mathbf{C}^{\times}$acts on the homogeneous coordinates with weights $\left(k_{1}, k_{2}, \ldots\right)$, exactly the same as for the weighted projective space. The local quotient singularities of the weighted projective space are replaced by stacky structures in the weighted projective stack.

As a trivial example, recall one of the presentations of the $G_{-1}^{k} \mathbf{P}^{N-1}$ gerbe is as a weighted projective stack, which could be called $W \mathbf{P}_{[k, k, \ldots, k]}^{N-1}$.

Traditionally, gauged linear sigma models with a single $U(1)$ and minimal charges $\left(k_{1}, k_{2}, \ldots\right)$ are believed to be associated to weighted projective spaces. However, the present work makes us suspect that, away from coupling extremes, such gauged linear sigma models might be more sensibly associated to weighted projective stacks, instead of spaces. One way to test this conjecture might be to examine quantum cohomology computations à la [3] and compare them to results obtained by other methods for ordinary weighted projective spaces (which must first be resolved before the quantum cohomology can be defined). We shall not speculate further on this subtle distinction of interpretations in this paper.

\subsection{Quantum cohomology for toric stacks}

There is now an easy prediction for part of the quantum cohomology of a toric stack. In [3], predictions for quantum cohomology of toric varieties were derived by computing one-loop corrections to effective actions in 
gauged linear sigma models. However, the authors of [3] were aware [8] of the physical distinction between gauged linear sigma models with minimal and nonminimal charges, and although they did not understand the mathematical interpretation, were careful to write down results also valid for gauged linear sigma models with nonminimal charges. Thus, on the face of it, the quantum cohomology calculations in [3] should also apply equally well to toric stacks, not just toric varieties. Indeed, we have checked that statement in previous sections by comparing several calculations of quantum cohomology for $\mathbf{Z}_{k}$ gerbes on projective spaces, some of those calculations coming from [3].

Now, we should qualify this statement slightly. We have argued in [1] that massless spectra should be computed by associated inertia stacks. Gauged linear sigma models, however, only seem to have direct UV access to the untwisted part of the associated inertia stack. Thus, one expects that the quantum cohomology predictions of [3] would not be predictions for the full quantum cohomology ring of a toric stack, but only part of it. Curiously, despite that expectation, the quantum cohomology rings we derived previously for $\mathbf{Z}_{k}$ gerbes over projective spaces were product structures on the cohomology of the entire inertia stack, not just one sector. Although the gauged linear sigma models only have direct UV access to part of the inertia stack, the quantum cohomology relations that one derives at least sometimes seem to know about all of the inertia stack. It is not clear whether that will be the case for all toric varieties, however.

In [9], a proposal was made for quantum cohomology rings of stacks. Their proposal was based on purely mathematical extrapolations of existing quantum cohomology calculations and made the assumption (not checked until [1]) that the right notion of massless spectrum should be given by inertia stacks. It would be interesting to compare their proposal for quantum cohomology of stacks to the physics results outlined in this paper.

\section{Calabi-Yau gerbes}

In [1], the notion of "Calabi-Yau" for stacks is discussed extensively, and it is argued that the "correct" notion is the naive one: a stack should be said to be Calabi-Yau if its canonical bundle is trivial. For Calabi-Yau gerbes, this constraint turns out to be rather trivial, as the canonical bundle of the gerbe is just a pullback of the canonical bundle of the underlying variety. Thus, a gerbe is Calabi-Yau if and only if the underlying variety is Calabi-Yau.

In this section, we shall discuss some Calabi-Yau gerbes. 


\subsection{Example: three-fold in $G_{-1}^{k} \mathbf{P}^{4}$ and Landau-Ginzburg orbifolds}

One easy way to construct some examples of gauged-linear-sigma-model presentations of gerbes over Calabi-Yau manifolds is to start with a gauged linear sigma model describing the Calabi-Yau and multiply the $U(1)$ charges of all fields by a constant, much as in the example discussed in Section 3.1. As already discussed, although perturbatively multiplying the charges of all the fields by a constant has no effect, nonperturbatively the resulting theory is different, so the full physical theory on a gerbe (for nonzero gauge coupling) is distinct from the sigma model on the underlying manifold.

For example, consider the quintic hypersurface in $\mathbf{P}^{4}$. As in [7], this is described by chiral superfields $x_{i}$ of charge 1 under a $U(1)$, together with a chiral superfield $p$ of charge -5 , and a superpotential of the form

$$
W=p G\left(x_{i}\right)
$$

where $G$ is the homogeneous polynomial of degree 5 defining the hypersurface. If we multiply the charges of all fields by $k$, so that the $x_{i}$ have charge $k$ and $p$ has charge $-5 k$, then the superpotential remains gauge-invariant, and the D-terms have the same form as before.

Clearly this process can be repeated for any Calabi-Yau, reflecting the fact discussed in [1] that all (Deligne-Mumford) gerbes over Calabi-Yau manifolds are themselves Calabi-Yau.

The phases of this gauged linear sigma model have the same general form as for the ordinary quintic. For large positive $r$, we recover a Calabi-Yau hypersurface in $G_{-1}^{k} \mathbf{P}^{4}$, which is a gerbe over the quintic.

For large negative $r$, we recover a Landau-Ginzburg orbifold phase. In this phase, $p \neq 0$, and its vacuum expectation value breaks the $U(1)$ to $\mathbf{Z}_{5 k}$. If we let $\xi$ denote a generator of the $(5 k)$ th roots of unity, then the residual gauge group action on the fields $x_{i}$ is generated by

$$
x_{i} \longmapsto \xi^{k} x_{i}
$$

for each $i$, since each $x_{i}$ has charge $k$. As a result, although the orbifold group is $\mathbf{Z}_{5 k}$, only $\mathbf{Z}_{5 k} / \mathbf{Z}_{k}=\mathbf{Z}_{5}$ acts effectively. Thus, the Landau-Ginzburg orbifold corresponding to this Calabi-Yau gerbe is a noneffective orbifold.

Orbifolds by noneffectively acting groups are discussed extensively in [1]. Although only the $\mathbf{Z}_{5}$ acts effectively, the physical theory is significantly different from the $\mathbf{Z}_{5}$ orbifold. In particular, we can now calculate the 
massless spectrum, and essentially because $\mathbf{Z}_{5 k}$ is abelian, we can immediately read off that the massless spectrum should be given by $k$ copies of the massless spectrum of the $\mathbf{Z}_{5}$ Landau-Ginzburg orbifold that corresponds to an ordinary quintic - for each twisted sector of the $\mathbf{Z}_{5}$ Landau-Ginzburg orbifold, we get $k$ copies in the present noneffective orbifold.

It is clear, in fact, that one will obtain results of a similar character for Landau-Ginzburg orbifolds corresponding to hypersurfaces in any weighted projective space.

Now, we can use this calculation to provide an important check of the closed string massless spectrum conjecture presented in [1]. There, we conjectured that the closed string massless spectrum of the IR fixed point of a gauged sigma model was given by the cohomology of the associated inertia stack. This agrees with standard results for orbifolds by finite effectively acting groups, and we argue extensively in [1] that this is also the correct result for orbifolds by finite noneffectively acting groups. However, for stacks that cannot be presented as quotients by finite groups, it is not currently possible to directly calculate the massless spectrum of the IR fixed point, as all presentations are as massive UV theories. Landau-Ginzburg orbifolds provide a workaround for this technical issue. Although the Landau-Ginzburg orbifold spectrum need not be precisely the same as that of the large-radius theory, in typical cases it is closely related.

Now, for this example of a $\mathbf{Z}_{k}$ gerbe over the quintic, the associated inertia stack is $k$ disjoint copies of the gerbe, and so the cohomology of the inertia stack is given by $k$ copies of the cohomology of the quintic. We computed above that the massless spectrum of the Landau-Ginzburg orbifold associated to our Calabi-Yau gerbe is given by $k$ copies of the massless spectrum of the Landau-Ginzburg $\mathbf{Z}_{5}$ orbifold associated to the ordinary quintic, in perfect agreement with the conjecture that massless spectra should be computed by the inertia stack. The same result follows for other easy examples of Calabi-Yau hypersurfaces. Thus, by computing spectra at LandauGinzburg points, we have obtained very strong evidence for the conjecture that massless spectra of strings on stacks should be counted by the inertia stack, in the case of stacks that cannot be presented as global quotients by finite groups.

\subsection{Example: Calabi-Yau in $G_{-n}^{k} \mathbf{P}^{N-1}$ and GLSM phases}

In the previous section, we discussed Calabi-Yau stacks built physically from gauged linear sigma models with nonminimal charges. As discussed earlier, we can also describe gerbes and stacks as e.g., $U(1)$ quotients of 
total spaces of principal $U(1)$ bundles. In this section, we shall outline the relevant physics of such descriptions.

Recall that banded $\mathbf{Z}_{k}$ gerbes over $\mathbf{P}^{N-1}$, i.e., the $G_{-n}^{k} \mathbf{P}^{N-1}$ model, can be described as a gauged linear sigma model with $N$ chiral superfields $x_{i}$ and one chiral superfield $z$ with charges under a pair of $U(1)$ 's as

\begin{tabular}{c|cc} 
& $x_{i}$ & $z$ \\
\hline$\lambda$ & 1 & $-n$ \\
$\mu$ & 0 & $k$
\end{tabular}

with D-terms constraining the $z$ to be nonzero. A Calabi-Yau hypersurface in such an object is $\left\{z G\left(x_{i}\right)=0\right\}$, where $G\left(x_{i}\right)$ is of degree $N$ in the $x_{i}$. Because of the D-term constraint, there is no new $\{z=0\}$ branch, the solutions of $z G\left(x_{i}\right)=0$ are the same as the solutions of $G\left(x_{i}\right)=0$. Clearly, for any Calabi-Yau presented as a hypersurface in a projective space, we can trivially construct a Calabi-Yau gerbe, in agreement with general observations [1] that a Calabi-Yau gerbe is merely a gerbe over a Calabi-Yau variety.

There is an obvious way to build a full gauged linear sigma model with a superpotential realizing the Calabi-Yau described above. Following the usual procedure, add a chiral superfield $p$ with charges $(n-N,-k)$ under $(\lambda, \mu)$. The superpotential of the theory is given by $p z G\left(x_{i}\right)$, and we have D-terms given by

$$
\begin{aligned}
\sum_{i}\left|x_{i}\right|^{2}-n|y|^{2}+(n-N)|p|^{2} & =r_{\lambda} \\
k|y|^{2}-k|p|^{2} & =r_{\mu}
\end{aligned}
$$

The analysis of the GLSM phases is straightforward. For $n \leq N$, we have a geometric phase where the Fayet-Iliopoulos terms are given by $r_{\lambda} \gg 0$, $r_{\mu} \gg 0$. In that regime, assuming a smooth hypersurface, $y \neq 0, p=0$, and not all the $x_{i}$ vanish.

However, we do not seem to ever have an ordinary Landau-Ginzburg phase in these models, merely hybrid Landau-Ginzburg phases at best. For example, when $r_{\lambda} \ll 0$ and $r_{\mu} \gg 0$, we have $y \neq 0$, and either $p=0$ (and some $x_{i}$ nonzero) or all of the $x_{i}=0$ (and $p \neq 0$ ). The second branch would correspond to a Landau-Ginzburg phase, but because of the first branch, this is not what one would ordinarily call an honest Landau-Ginzburg point.

Also note that if $n>N$, then we do not seem to have a purely geometric phase any longer. In the regime $r_{\lambda} \gg 0$, we are merely guaranteed that not all of the $\left\{x_{i}, p\right\}$ vanish. Thus, in this phase, we have a new branch. 


\section{Mirrors to stacks}

In this section, we will discuss how gauged-linear-sigma-model-based methods of $[6,10]$ for computing mirrors can be applied to the gauged linear sigma model descriptions of gerbes discussed in this paper. We will begin with the A model on gerbes on projective spaces and derive corresponding Toda theories and then discuss the mirror of strings on gerbes on quintic three-folds.

\subsection{Toda theories corresponding to gerbes on projective spaces}

For simplicity, we shall begin with gerbes on $\mathbf{P}^{1}$ and shall derive Toda theories from several presentations. Then, we shall briefly outline gerbes on more general projective spaces.

\subsubsection{Toda theory for $G_{-1}^{k} \mathbf{P}^{N-1}$}

Recall that the $G_{-1}^{k} \mathbf{P}^{N-1}$ model is described by $N$ chiral superfields $x_{i}$, each of charge $k$ with respect to a gauged $U(1)$. In other words, it can be presented identically to the $\mathbf{C P}^{N-1}$ model, except that the gauge charges are minimal.

Following the prescription of $[6,10]$, the "mirror" theory should be described by $N$ neutral chiral superfields $Y_{i}$, with one gauge multiplet $\Sigma$, and an effective superpotential

$$
\widetilde{W}=\Sigma\left(k Y_{1}+\cdots+k Y_{N}\right)+\sum_{i=1}^{N} \exp \left(-Y_{i}\right)
$$

where we are being sloppy about FI terms and factors, which will not play an essential role in our discussion. Integrating out the $Y_{i}$ yields an effective superpotential for $\Sigma$ identical to that obtained by one-loop calculations in [3]. If instead we integrate out $\Sigma$, then in principle we are left with an effective Landau-Ginzburg theory whose B model correlators should match the A model correlation functions of the $G_{-1}^{k} \mathbf{P}^{N-1}$ model.

Integrating out $\Sigma$ is slightly subtle. It gives the constraint

$$
k\left(Y_{1}+\cdots+Y_{N}\right)=0
$$

Because the $Y_{i}$ are periodic, it is not quite right to divide out the $k$. Rather, this constraint says that $\exp \left(-Y_{N}\right)$ only matches

$$
\exp \left(Y_{1}\right) \exp \left(Y_{2}\right) \cdots \exp \left(Y_{N-1}\right)
$$


up to a $k$ th root of unity, call it $\Upsilon$. Thus, integrating out $\Sigma$ gives us an effective superpotential

$$
\widetilde{W}=\exp \left(-Y_{1}\right)+\cdots+\exp \left(-Y_{N-1}\right)+\Upsilon \prod_{i=1}^{N-1} \exp \left(Y_{i}\right)
$$

We will derive this same result from an alternative presentation of the gerbe in the next section.

\subsubsection{Toda theory for $G_{-n}^{k} \mathbf{P}^{N-1}$}

Recall that the $G_{-n}^{k} \mathbf{P}^{N-1}$ models are described by a gauged linear sigma model with $N$ chiral superfields $x_{i}$ and one chiral superfield $z$, charged with respect to a pair of $U(1)$ gauge symmetries as follows:

\begin{tabular}{c|cc} 
& $x_{i}$ & $z$ \\
\hline$\lambda$ & 1 & $-n$ \\
$\mu$ & 0 & $k$
\end{tabular}

Following the prescription of $[6,10]$, the "mirror" theory can be obtained from a theory with neutral chiral superfields $Y_{1}, Y_{2}, \ldots, Y_{N}, Y_{z}$, corresponding to the $x_{i}$ and $z$ chiral superfields, and two gauge multiplets $\Sigma_{\lambda}, \Sigma_{\mu}$, with a superpotential ${ }^{5}$

$$
\widetilde{W}=\Sigma_{\lambda}\left(Y_{1}+\cdots Y_{N}-n Y_{z}\right)+\Sigma_{\mu}\left(k Y_{z}\right)+\sum_{i=1}^{N} \exp \left(-Y_{i}\right)+\exp \left(-Y_{z}\right)
$$

Integrating out the $Y$ 's returns an effective superpotential for the $\Sigma$ 's identical to that calculated from one-loop effects in [3]. Integrating out the $\Sigma$ 's gives the constraints ${ }^{6}$

$$
\begin{aligned}
Y_{1}+\cdots+Y_{N}-n Y_{z} & =0 \\
k Y_{z} & =0
\end{aligned}
$$

and an effective superpotential

$$
\begin{aligned}
\tilde{W}= & \exp \left(-Y_{1}\right)+\cdots+\exp \left(-Y_{N-1}\right) \\
& +\exp \left(Y_{1}\right) \exp \left(Y_{2}\right) \cdots \exp \left(Y_{N-1}\right) \Upsilon^{-n}+\Upsilon
\end{aligned}
$$

where $\Upsilon=\exp \left(Y_{z}\right)$, and hence is constrained by $\Upsilon^{k}=1$, and where we have eliminated the field $Y_{N}$ using the other constraint equation. (Note that

\footnotetext{
${ }^{5}$ We are being sloppy about FI terms and scales. A meticulous reader will find it trivially easy to reinsert both in our expressions.

${ }^{6}$ The two constraints below would be the D-term equations, if we had included FI terms above.
} 
since the $Y$ 's are periodic, the constraint $k Y_{z}=0$ does not eliminate $Y_{z}$, but rather merely forces it to define a $k$ th root of unity.)

This new theory, with $N-1$ neutral chiral superfields $Y_{1}, \ldots, Y_{N-1}$, together with a $\mathbf{Z}_{k}$-valued field $\Upsilon$, and the effective superpotential above, is our proposed Toda mirror to the $G_{-n}^{k} \mathbf{P}^{N-1}$ model in the sense that A model correlation functions of the latter can be calculated as B model correlation functions of the former. We will show in the next section that correlation functions do indeed match.

In passing, note that for $n=1$, this theory specializes to our earlier result for the first presentation of the $G_{-1}^{k} \mathbf{P}^{N-1}$ model.

\subsubsection{Check of correlation functions of Toda duals}

To check that the Toda theories just described really do correspond to the gerby $\mathbf{P}^{N-1}$ models $G_{-n}^{k} \mathbf{P}^{N-1}$, we shall compute the B model correlation functions of the Toda theory and check that they match the A model correlation functions of the gerby $\mathbf{P}^{N-1}$ models.

In fact, let us back up one step further and first review how B model periods are calculated in the Toda theory corresponding to the ordinary $\mathbf{C P}^{N-1}$ model.

Recall from [11] that given a B-twisted Landau-Ginzburg model with superpotential $W$, if we define $H=\operatorname{det}\left(\partial_{i} \partial_{j} W\right)$, then the tree-level correlation functions can be calculated in the form

$$
<F_{1} \cdots F_{n}>=\sum_{d W=0} \frac{F_{1} \cdots F_{n}}{H}
$$

where the $F_{i}$ are polynomials in the chiral superfields, i.e., observables of the B-twisted Landau-Ginzburg model.

The Toda theory corresponding to the ordinary $\mathbf{P}^{N-1}$ model is a B-twisted Landau-Ginzburg model with superpotential

$$
W=\exp \left(Y_{1}\right)+\cdots+\exp \left(Y_{N-1}\right)+\exp \left(-Y_{1}-Y_{2}-\cdots-Y_{N-1}\right)
$$

Define $\Pi=\exp \left(-Y_{1}-Y_{2}-\cdots-Y_{N-1}\right)$; then the classical vacua are defined by

$$
\exp \left(Y_{1}\right)=\cdots=\exp \left(Y_{N-1}\right)=\Pi
$$

which implies that

$$
\left(\exp \left(Y_{i}\right)\right)^{N}=1
$$

for all $i$. When restricted to classical vacua, it is straightforward to compute that the determinant $H=N \Pi^{N-1}$. Thus, if we define $X=\exp \left(Y_{1}\right)$, then 
for correlation functions we compute that

$$
<X^{m}>=\sum \frac{X^{m}}{N X^{N-1}}
$$

using the fact that at classical vacua, $X=\Pi$, and where the sum runs over $N$ th roots of unity. This expression can only be nonvanishing when $m+1-N$ is divisible by $N$, and thus the only nonvanishing correlation functions are

$$
<X^{N-1}>,<X^{2 N-1}>,<X^{3 N-1}>, \cdots
$$

By comparison, recall that the ordinary $\mathbf{P}^{N-1}$ model has quantum cohomology relation $X^{N}=q$ and classical correlation function $\left\langle X^{N-1}\right\rangle=1$, from which one computes that the nonvanishing correlation functions, beyond the classical correlation function, are

$$
\begin{aligned}
& <X^{2 N-1}>=q \\
& <X^{3 N-1}>=q^{2}
\end{aligned}
$$

and so forth, in agreement with the Toda calculation above.

Now let us turn to the proposed Toda dual to the $G_{-n}^{k} \mathbf{P}^{N-1}$ model. Recall that this Toda theory has $N-1$ neutral chiral superfields $Y_{i}$, together with a $\mathbf{Z}_{k}$-valued field $\Upsilon$, and the effective superpotential (6.1). It is very straightforward to generalize the methods of [11] to this situation. Since the $\mathbf{Z}_{k}$-valued fields are annihilated by supersymmetry transformations, and so do not have superpartners, we can immediately deduce:

- Each $\mathbf{Z}_{k}$-valued field is itself a BRST-invariant observable.

- As the Hessian factor is derived from F-terms and Yukawa couplings, in a theory with $\mathbf{Z}_{k}$-valued fields, it is calculated only for complex-valued chiral superfields, omitting the $\mathbf{Z}_{k}$-valued fields.

Otherwise the form of the calculations in [11] are unchanged. Thus, following [11], tree-level correlators have the form

$$
<F_{1} \cdots F_{p}>=\sum_{d W=0} \frac{F_{1} \cdots F_{p}}{H}
$$

where $H$ is the determinant of the matrix of second derivatives of the superpotential, taking derivatives corresponding to complex-valued chiral superfields, and the $F$ 's are combinations of $\exp \left(Y_{i}\right)$ and $\Upsilon$. The solutions of 
$d W=0$ are given by

$$
\exp \left(-Y_{i}\right)=\exp \left(Y_{1}\right) \exp \left(Y_{2}\right) \cdots \exp \left(Y_{N-1}\right) \Upsilon^{-n}
$$

for all $i$, and as $\Upsilon$ is $\mathbf{Z}_{k}$-valued, all its values trivially satisfy $d W=0$. Let us define

$$
\Pi=\exp \left(Y_{1}\right) \exp \left(Y_{2}\right) \cdots \exp \left(Y_{N-1}\right) \Upsilon^{-n}
$$

so that the classical vacua can be described as

$$
\exp \left(-Y_{1}\right)=\exp \left(-Y_{2}\right)=\cdots=\exp \left(-Y_{N-1}\right)=\Pi
$$

Then, on the locus of classical vacua, it can be shown that

$$
H=N \Pi^{N-1}
$$

Finally, let us define $X=\exp \left(-Y_{1}\right)$, which from the work above we see satisfies $X^{N}=\Upsilon^{-n}$ on the locus of classical vacua.

For reasons already outlined, correlation functions in this theory can then be described as

$$
\begin{aligned}
<X^{m} \Upsilon^{p}> & =\sum_{d W=0} \frac{X^{m} \Upsilon^{p}}{H} \\
& =\sum_{\Upsilon \in \mathbf{Z}_{k}} \sum_{X^{N}=\Upsilon^{-n}} \frac{X^{m} \Upsilon^{p}}{N X^{N-1}}
\end{aligned}
$$

This sum will only be nonvanishing when

$$
m-N+1=r N
$$

for some integer $r$ (taking advantage of $X^{N}=\Upsilon^{-n}$ ) such that $p-n r=s k$ for some integer $s$ (taking advantage of $\Upsilon^{k}=1$ ). For example, if

$$
m-N+1=r N+\epsilon
$$

for some integer $\epsilon$ between 0 and $N$, then the correlation function has an internal sum of the form $\sum_{X} X^{\epsilon}$ over roots $X$ on the classical locus, which vanishes.

In any event, we see that the nonvanishing correlation functions in this theory are of the form

$$
<X^{N(r+1)-1} \Upsilon^{n r+s k}>
$$

for integers $r, s$, which exactly matches our earlier result, equation (3.1) in Section 3.3, for A model correlation functions in the $G_{-n}^{k} \mathbf{P}^{N-1}$ model, if we identify $Y \sim \Upsilon^{-1}$.

Let us take a moment to discuss some alternative potential Toda theories corresponding to the $G_{-1}^{k} \mathbf{P}^{N-1}$ model and reasons why they are not 
acceptable. For example, for the $G_{-1}^{k} \mathbf{P}^{1}$ model, one natural guess for a corresponding Toda theory would have superpotential

$$
W=\exp (k \Theta)+\exp (-k \Theta)
$$

just as the ordinary $\mathbf{P}^{1}$ model has corresponding Toda theory with superpotential

$$
W=\exp (\Theta)+\exp (-\Theta)
$$

However, the periods of this Toda theory do not reproduce the A model quantum cohomology calculations discussed earlier. Define $X=\exp (\Theta)$; then following [11]

$$
<X^{m}>=\sum_{d W=0} \frac{X^{m}}{H}
$$

In the present case,

$$
H=k^{2}(\exp (k \Theta)+\exp (-k \Theta))=k^{2}\left(X^{k}+X^{-k}\right)
$$

and the classical vacua are defined by $X^{k}=X^{-k}$ or $X^{2 k}=1$. Thus, the classical vacua are the $2 k$ th roots of unity, so we do at least have as many classical vacua as expected. The B model correlation functions, however, are more problematic:

$$
<X^{m}>\propto \sum X^{m-k}
$$

where the sum is over $2 k$ th roots of unity, and hence is only nonvanishing when $m-k$ is a multiple of $2 k$, which implies that the nonzero correlation functions are

$$
<X^{k}>,<X^{3 k}>,<X^{5 k}>, \ldots
$$

However, previously we have calculated for this model that the nonvanishing correlation functions are given by

$$
<X>=1,<X^{2 k+1}>=q,<X^{4 k+1}>=q^{2}, \ldots
$$

which does not match the result above, except in the trivial case $k=1$.

Furthermore, the obvious extension of the alternate proposal above to $G_{-1}^{k} \mathbf{P}^{N-1}$, namely the Toda theory with superpotential

$$
W=\exp \left(k Y_{1}\right)+\cdots+\exp \left(k Y_{N-1}\right)+\exp \left(-k Y_{1}-k Y_{2}-\cdots-k Y_{N-1}\right)
$$

not only does not have matching correlation functions, but does not even have the expected number of classical vacua (it has $k N(N-1)$ instead of $k N)$. 


\subsection{Mirrors to gerbes on the quintic}

Let us begin our discussion of mirrors to gerbes over the quintic by appealing to a simple minimal-model argument at the Fermat Landau-Ginzburg point in the moduli space. As noticed previously in Section 5.1, the LandauGinzburg orbifold corresponding to the quintic hypersurface in $G_{-1}^{k} \mathbf{P}^{N-1}$ is a noneffectively acting $\mathbf{Z}_{5 k}$ orbifold. Thus, at the Fermat point, the theory can be constructed as a $\mathbf{Z}_{5 k}$ orbifold of a tensor product of five minimal models, call them each $M$ :

$$
\left[\frac{M \otimes M \otimes \cdots \otimes M}{\mathbf{Z}_{5 k}}\right]
$$

Now, by ordinary mirror symmetry for the minimal models, we can replace each $M$ by the $\mathbf{Z}_{5}$ orbifold $\left[M / \mathbf{Z}_{5}\right]$, leaving us with a $\mathbf{Z}_{5 k}$ orbifold of a tensor product of five copies of $\left[M / \mathbf{Z}_{5}\right]$. As shown in [4], a noneffective $\mathbf{Z}_{k n}$ orbifold of a $\mathbf{Z}_{n}$ orbifold is a trivially acting $\mathbf{Z}_{k}$ orbifold. Thus, in the present case, we are left with a trivially acting $\mathbf{Z}_{k}$ orbifold of a $\mathbf{Z}_{5}^{4}$ orbifold of a tensor product of five minimal models, i.e., a trivially acting $\mathbf{Z}_{k}$ orbifold of the mirror to the standard Landau-Ginzburg Fermat model.

In light of the result [4] that the mirror to a trivial gerbe is a trivial gerbe over the mirror, the calculation above implies that, at the Fermat Landau-Ginzburg point, the conformal field theory of the $\mathbf{Z}_{5 k}$ orbifold is the same as a $\mathbf{Z}_{5} \times \mathbf{Z}_{k}$ orbifold. Note that this statement is consistent with partition functions: since $\mathbf{Z}_{5 k}$ is abelian, the $g$-loop partition function of the $\mathbf{Z}_{5 k}$ orbifold is the same as that of the $\mathbf{Z}_{5} \times \mathbf{Z}_{k}$ orbifold, for all $g$.

Let us now formally compute the mirror of the gerby quintic using the methods of $[6,10,12]$. Since this is a hypersurface in a toric variety, and not a toric variety, we shall use the trick of [12] of replacing the A model on such a hypersurface with the A model on a toric supervariety, which can be dualized using the methods of $[6,10]$ to an effective Landau-Ginzburg theory whose B model correlation functions should duplicate the original A model correlation functions. (Unfortunately, this is a many-to-one map, as complex structure data is lost on the A side, corresponding to the fact that the dual is fixed at one point in Kähler moduli space. Nevertheless, this will give good insight into the structure of the mirrors.)

Following [6], let us first compute the mirror of the ambient gauged linear sigma model with no superpotential. The mirror can be described starting from a theory with neutral chiral superfields $Y_{1}, \ldots, Y_{5}, Y_{p}$ and $\Sigma$, and effective superpotential

$$
\widetilde{W}=\Sigma\left(-5 k Y_{p}+k Y_{1}+\cdots+k Y_{5}-k r\right)+\sum_{i} \exp \left(-Y_{i}\right)+\exp \left(-Y_{p}\right)
$$


where $r$ is proportional to the Fayet-Iliopoulos term. (We have included it here, with a suitable normalization, to make the result cleaner.) For simplicity, we will assume that $k$ is not divisible by 5 . Integrating out $\Sigma$ gives us the constraint

$$
k Y_{p}=-\frac{1}{5} k\left(Y_{1}+\cdots+Y_{5}-r\right)
$$

Since the fields $Y$ are periodic, if we define $X_{i}=\exp \left(-Y_{i} / 5\right)$, then we have that

$$
\exp \left(-Y_{p}\right)=\Upsilon \exp (-r / 5) X_{1} X_{2} X_{3} X_{4} X_{5}
$$

where $\Upsilon$ is a $k$ th root of unity, whose possible values must be summed over in the path integral measure. Furthermore, because $X_{i}=\exp \left(-Y_{i} / 5\right)$, we have identifications

$$
X_{i} \sim \omega_{i} X_{i}
$$

where $\omega_{i}$ is a fifth root of unity. Using the assumption that 5 does not divide $k$, the relation between $Y_{p}$ and the $Y_{i}$ tells us that $\prod_{i} \omega_{i}=1$, so in fact we must orbifold by $\mathbf{Z}_{5}^{4}$ rather than $\mathbf{Z}_{5}^{5}$. Thus, we have an effective LandauGinzburg model of the mirror defined by the effective superpotential

$$
\widetilde{W}=X_{1}^{5}+\cdots+X_{5}^{5}+\Upsilon \exp \left(-\frac{r}{5}\right) X_{1} X_{2} X_{3} X_{4} X_{5}
$$

and the $\mathbf{Z}_{5}^{4}$ orbifold action described previously, where $\Upsilon$ is a $k$ th root of unity, and the path integral measure must sum over values of $\Upsilon$. So far we have only computed the mirror of the gauged linear sigma model with vanishing superpotential. In this theory, the fundamental fields ${ }^{7}$ over which the path integral measure sums are the $Y$ 's, not the $X$ 's. It is easy to check that turning on a superpotential, and proceeding as in [12], has the ultimate effect only of changing the fundamental fields from the $Y$ 's to the $X$ 's. Otherwise, just as for the quintic in ordinary $\mathbf{P}^{4}$, the form of the effective Landau-Ginzburg theory is the same between the two cases. In particular, the effective Landau-Ginzburg theory dual to the A model on the gerby quintic is defined by the superpotential above, with the field $\Upsilon$ taking values in roots of unity.

At the Fermat point, where the $\prod_{i} X_{i}$ term decouples, the theory reduces to a $\mathbf{Z}_{5}^{4}$ orbifold of a product of five minimal models, tensored with the theory of the $\mathbf{Z}_{k}$-valued field $\Upsilon$. However, as discussed in [4], tensoring with the

\footnotetext{
${ }^{7}$ As a result, for example, the untwisted part of the chiral ring in the dual to the theory with no superpotential is given by the invariant part of $\mathbf{C}\left[Y_{1}, Y_{2}, \ldots, Y_{5}\right] /(\partial W)$ with $W$ of the form above, an infinite-dimensional vector space, reflecting the noncompactness of the original theory. Turning on the superpotential in the original theory yields a dual with chiral ring untwisted sector given by the invariant part of $\mathbf{C}\left[X_{1}, X_{2}, \ldots, X_{5}\right] /(\partial W)$, a finite-dimensional vector space. We would like to thank A. Adams for discussions of these matters.
} 
trivial theory of a $\mathbf{Z}_{k}$-valued field is equivalent to orbifolding by a trivially acting $\mathbf{Z}_{k}$, so we recover the same result at the Fermat point that we derived earlier using nothing more than standard facts about minimal models.

Away from the Fermat point, we see something interesting. The $\mathbf{Z}_{k}$-valued field $\Upsilon$, which at Fermat merely realizes a trivially acting $\mathbf{Z}_{k}$ orbifold, now mixes nontrivially with super-potential terms. Recall that the same sort of structure appeared from an independent line of thought in [4] when discussing the interpretation of moduli in twisted sectors associated to trivially acting group elements. In hindsight, this should not be surprising - both here and in [4], we are ultimately studying the same sorts of problems, namely deformations of stringy moduli appearing in gerbes. Thus, we should not be surprised to see the same structures emerging in both cases.

\subsection{Gerby minimal models}

Let us take just a moment to outline some easy results concerning mirror symmetry and noneffective orbifolds of minimal models.

Consider the $A_{d-1}$ minimal model, which arises as the IR fixed point of the LG model of a single chiral superfield $\Phi$ with superpotential $\Phi^{d}$. It is well known that model is mirror to its orbifold by $\mathbf{Z}_{d}$, acting as $\Phi \mapsto \exp (2 \pi i / d) \Phi$. We can generate an easy example of gerby mirrors in minimal models using the result in [4] that for any $\mathrm{CFT} \mathcal{C}$, if we define $\mathcal{C}^{\prime}$ to be the orbifold $\left[\mathbf{C} / \mathbf{Z}_{d}\right]$, then the orbifold $\left[\mathbf{C}^{\prime} / \mathbf{Z}_{d k}\right]$ (where $\mathbf{Z}_{d}$ is the quantum symmetry and $\mathbf{Z}_{k}$ acts trivially) is isomorphic to the orbifold $\left[\mathcal{C} / \mathbf{Z}_{k}\right]$. Applied to the minimal model above, this means that the $\mathbf{Z}_{d k}$ orbifold of the $A_{d-1}$ minimal model (where the $\mathbf{Z}_{d k}$ acts by projecting to $\mathbf{Z}_{d}$, acting in the usual way, with trivial $\mathbf{Z}_{k}$ kernel) is isomorphic to the trivial $\mathbf{Z}_{k}$ orbifold of the same minimal model.

Furthermore, from the argument in [4] that trivial gerbes are mirror, one expects that the trivial $\left[A_{d-1} / \mathbf{Z}_{k}\right]$ orbifold should be mirror to $\left[\left[A_{d-1} / \mathbf{Z}_{d}\right] /\right.$ $\left.\mathbf{Z}_{k}\right] \cong\left[A_{d-1} /\left(\mathbf{Z}_{d} \oplus \mathbf{Z}_{k}\right)\right]$, hence the $\mathbf{Z}_{k d}$ and $\mathbf{Z}_{d} \oplus \mathbf{Z}_{k}$ orbifolds of the $A_{d-1}$ minimal model should be isomorphic. It is trivial to check that spectra and $g$-loop partition functions match between two such orbifolds, hence the result seems very plausible.

\subsection{Analogue of Batyrev's mirror conjecture for stacks}

In order to find an analogue of Batyrev's mirror conjecture [13], one faces the following basic puzzle: since a toric stack is described in terms of a fan 
plus finite-group data associated to each edge (see the appendix for more information), in order for reflexive polyhedra to encode mirrors, Newton polygons must somehow be decorated with the same sort of finite group data that appears in toric stacks.

This puzzle is related to the puzzle posed by deformation theory of stacks: the only mathematical deformations of a stack are those in the untwisted sector. Twist field moduli do not have a purely mathematical understanding.

We have seen how explicit mirror constructions solve the deformation theory puzzle, by forcing us to introduce a new class of abstract CFTs, based on Landau-Ginzburg orbifolds, in which the superpotential is deformed by finite-group-valued fields, corresponding to giving a vev to a twist field in a noneffective orbifold.

The solution to the puzzle in generalizing Batyrev's conjecture to toric stacks is the same: we need to consider the same general class of abstract CFTs, in which superpotentials naturally include monomial terms with finite-group factors.

It is now straightforward to generalize Batyrev's conjecture. Recall that in its ordinary form, given a Calabi-Yau hypersurface in a toric variety, one first constructs the Newton polygon of possible monomial contributions to the hypersurface and another polygon with faces over the cone of the fan describing the ambient toric variety. (One typically scales so that each polyhedron, on an integral lattice, has exactly one interior point.) So long as these polyhedra are reflexive, the mirror is obtained by exchanging these polyhedra: the mirror ambient toric variety has fan defined by the Newton polyhedron of the original, and the monomials in a Calabi-Yau hypersurface are described by the polyhedron over the fan of the original ambient toric variety. (See [14] for a very readable discussion.)

To generalize this procedure to stacks, we proceed as follows. The stacky fan of the mirror ambient toric variety is obtained by using the above construction to get the basic fan, with finite-group data obtained by taking the difference of finite-group factors between the monomial corresponding to that corner of the polyhedron and the monomial corresponding to the interior point. The Newton polyhedron of the mirror theory is obtained from the same procedure as for the standard construction, with finite-groupvalued-field-factors obtained from the generators decorating each edge of the stacky fan.

Let us work through a simple example, to make this process clearer. Let us first review Batyrev's mirror construction for elliptic curves, built as degree three hypersurfaces in $\mathbf{P}^{2}$, and then we shall describe how to generalize this 
process to describe the mirror of the gerbe over an elliptic curve obtained by restricting the $G_{-1}^{k} \mathbf{P}^{2}$ model to the hypersurface.

In figure 1, we have shown the fan for the toric variety $\mathbf{P}^{2}$. In figure 2 , we have shown the Newton polyhedron for degree three hypersurfaces in $\mathbf{P}^{2}$.

The fan of the mirror ambient toric variety is obtained as the fan through the faces of the Newton polyhedron and is shown in figure 2. It is straightforward to compute that this fan describes the toric variety $\mathbf{P}^{2} / \mathbf{Z}_{3}$, where if we denote the homogeneous coordinates on $\mathbf{P}^{2}$ by $\left[z_{0}, z_{1}, z_{2}\right]$, and let $\xi$ generate the third roots of unity, then the generator of the $\mathbf{Z}_{3}$ acts as

$$
\left[z_{0}, z_{1}, z_{2}\right] \longmapsto\left[z_{0}, \xi z_{1}, \xi^{2} z_{2}\right]
$$

In a little more detail, if we let the three cones in the fan be denoted I, II, III, where I is the cone covering the first quadrant and the others proceed counterclockwise, then the coordinate patch corresponding to cone I is given by

$$
\text { Spec } \mathbf{C}\left[x^{2} y, x y^{2}, x y\right]
$$

the coordinate patch corresponding to cone II is given by

$$
\operatorname{Spec} \mathbf{C}\left[x^{-2} y^{-1}, x^{-1} y, x^{-1}\right]
$$

and the coordinate patch corresponding to cone III is given by

$$
\text { Spec } \mathbf{C}\left[x y^{-1}, x^{-1} y^{-2}, y^{-1}\right]
$$

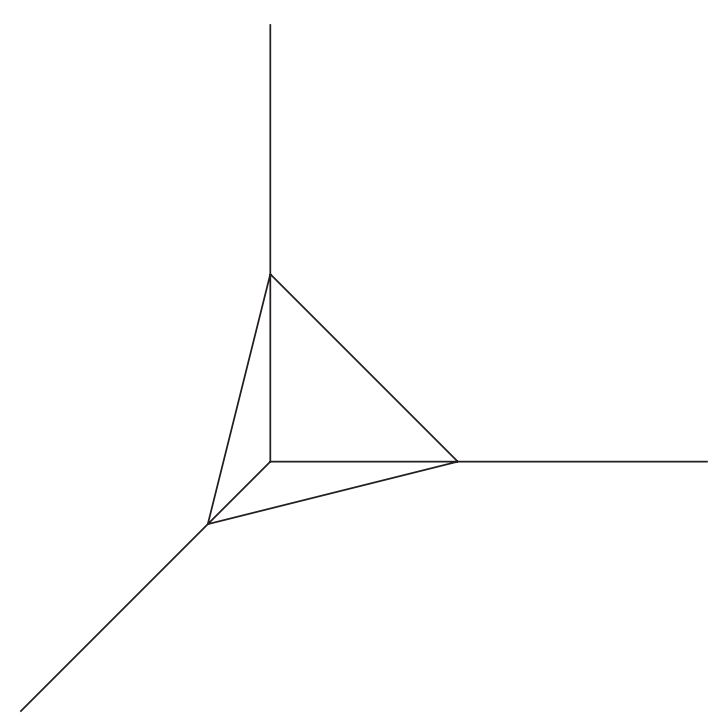

Figure 1: The fan for $\mathbf{P}^{2}$, with the mirror Newton polyhedron shown. 


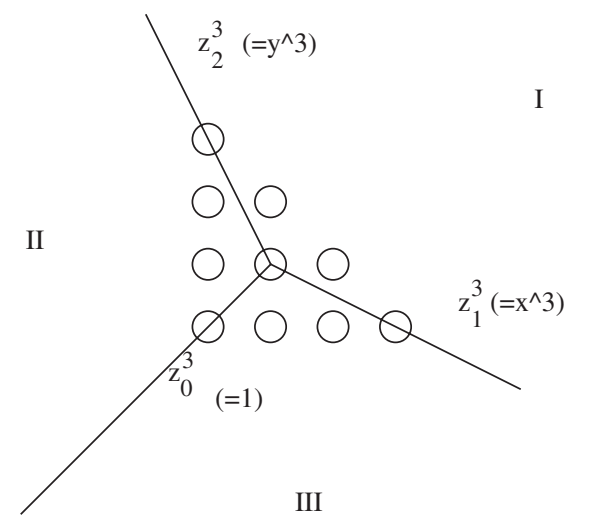

Figure 2: The Newton polyhedron for degree three hypersurfaces in $\mathbf{P}^{2}$. The three corner points, corresponding to cubes of the three homogeneous coordinates, are labelled. The fan of the mirror ambient toric variety, the fan over the faces of the Newton polyhedron, is shown.

We can relate this description to the homogeneous coordinates on $\mathbf{P}^{2}$ by identifying

$$
x \sim \frac{z_{1}^{2}}{z_{0} z_{2}}, \quad y \sim \frac{z_{2}^{2}}{z_{0} z_{1}}
$$

The Newton polyhedron for degree three hypersurfaces in $\mathbf{P}^{2} / \mathbf{Z}_{3}$ has monomials

$$
\left\{z_{0}^{3}, z_{1}^{3}, z_{2}^{3}, z_{0} z_{1} z_{2}\right\}=\left\{1, x^{2} y, x y^{2}, x y\right\}
$$

and is shown in figure 3 .

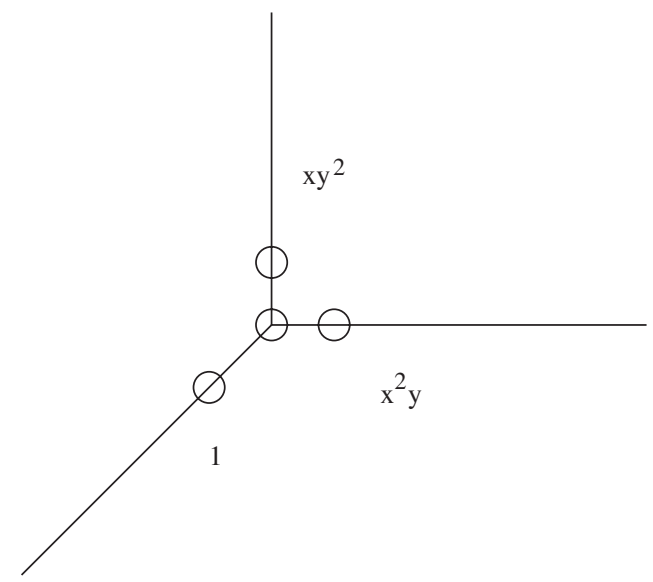

Figure 3: Newton polyhedron for degree three hypersurfaces in $\mathbf{P}^{2} / \mathbf{Z}_{3}$, with fan over the faces shown. 
Note that the fan shown in figure 3, constructed from the mirror Newton polyhedron, is the same as the fan of the original $\mathbf{P}^{2}$, so that applying the mirror construction twice returns to the original toric variety and hypersurface.

Now that we have seen how Batyrev's construction works for the case of elliptic curves in $\mathbf{P}^{2}$, let us turn to a simple example of a toric stack. Consider the $\mathbf{Z}_{k}$ gerbe over $\mathbf{P}^{2}$ with characteristic class $-1 \bmod k$. As described in more detail in the appendix, the stacky fan has underlying fan given by the same fan as $\mathbf{P}^{2}$, and to the edges we associate generators of $\mathbf{Z}^{2} \oplus \mathbf{Z}_{k}$, given by

$$
\begin{gathered}
(1,0,0) \\
(0,1,0) \\
(-1,-1,1)
\end{gathered}
$$

If we consider gerbes over ordinary elliptic curves, so that the possible monomials do not have any finite-group factors, then the mirror ambient toric stack has fan given by that for $\mathbf{P}^{2} / \mathbf{Z}_{3}$, and to the edges we associate generators of $\mathbf{Z}^{2} \oplus \mathbf{Z}_{k}$ given by

$$
\begin{gathered}
(-1,2,0) \\
(2,-1,0) \\
(-1,-1,0)
\end{gathered}
$$

Since there are no finite-group factors in the monomials, the generators of $\mathbf{Z}^{2} \oplus \mathbf{Z}_{k}$ do not extend into $\mathbf{Z}_{k}$. The Newton polygon of the mirror is constructed from the polygon over the fan of the ambient toric stack. The possible monomials are weighted by finite-group data, in a fashion determined by the generators (6.2) of the original toric stack. Specifically, we have monomial terms

$$
\left\{\Upsilon z_{0}^{3}, z_{1}^{3}, z_{2}^{3}, z_{0} z_{1} z_{2}\right\}
$$

in the superpotential of the mirror Landau-Ginzburg theory, where $\Upsilon$ is a $\mathbf{Z}_{k}$-valued field, which appears in the $z_{0}^{3}$ monomial because the generator associated to the corresponding edge of the toric fan extends into $\mathbf{Z}_{k}$. If the generators associated to the edges had been

$$
\begin{gathered}
(1,0, a) \\
(0,1, b) \\
(-1,-1, c)
\end{gathered}
$$

then the monomial terms in the superpotential of the mirror LandauGinzburg model would have been

$$
\left\{\Upsilon^{c} z_{0}^{3}, \Upsilon^{a} z_{1}^{3}, \Upsilon^{b} z_{2}^{3}, z_{0} z_{1} z_{2}\right\}
$$

Similarly, if there were additional finite-group factors in the defining data of the stacky fan, there would be additional finite-group-valued fields appearing in the mirror Landau-Ginzburg superpotentials. 
Also note that if we work with a stacky fan with $\mathbf{Z}_{k}$ factors but no generator extends into those factors, and the superpotential has only ordinary monomials - no finite-group-valued field factors - then the mirror of the stack is the mirror Calabi-Yau, in the ordinary sense, in a stacky fan with $\mathbf{Z}_{k}$ factors. This can be interpreted as the statement that the mirror of the trivial $\mathbf{Z}_{k}$-gerbe over a Calabi-Yau is the trivial $\mathbf{Z}_{k}$-gerbe over the mirror Calabi-Yau, agreeing with easy general statements on mirrors of trivial gerbes described in $[1,4]$.

In passing, note that the monomial-divisor mirror map now immediately generalizes to toric stacks, as we have implicitly constructed a map between generators of the Kähler-like deformations of the theories with finite-groupvalued fields, and the complex-structure-like deformations, in the same form as the original monomial-divisor mirror map.

In order to properly understand whether this proposed generalization of Batyrev's mirror conjecture is physically correct, we would need to further pursue properties of these new abstract CFTs constructed from LandauGinzburg orbifolds with finite-group-valued fields. We will leave such a study for future work, and for the purposes of this paper, content ourselves with having merely elucidated the conjecture.

\section{Conclusions}

In this paper, we have discussed gauged linear sigma models with nonminimal $U(1)$ charges. Such theories, which describe quotients by noneffectively acting $\mathbf{C}^{\times}$'s, are physically distinct from their counterparts with minimal charges (as we saw in the analogous case of finite noneffectively acting groups in [4]), and can be understood mathematically as describing toric stacks.

Every stack has a presentation of the form $[X / G]$, to which one associates a $G$-gauged sigma model on $X$. However, such presentations are not unique. We have argued in [1] and seen further examples here showing that stacks classify universality classes of worldsheet RG flow in gauged sigma models. Curiously, this seems to be true not only for Calabi-Yau stacks, but also for the A model on more general stacks, as we have seen in examples here.

We have discussed quantum cohomology of gauged linear sigma models corresponding to toric stacks, as well as massless spectra of LandauGinzburg models associated to gerby hypersurfaces, and seen how the results in all such cases are consistent with the general conjecture for massless spectra in gauged sigma models presented in [1], namely that the massless spectrum is the de Rham cohomology of the associated inertia stack. 
Finally, and perhaps most importantly, we have discussed mirror symmetry for stacks and seen how fields valued in roots of unity play an important role, in Toda duals to toric stacks, in the mirror to the gerby quintic, and more generally in formulating Batyrev's mirror conjecture for hypersurfaces in toric stacks. Previously in $[1,4]$, we have seen such fields appearing when understanding deformations along marginal operators in certain twisted sectors of noneffective orbifolds and used them to give a very explicit understanding of the CFTs one obtains by such deformations. Here, although fields valued in roots of unity are derived from completely independent lines of reasoning, the root cause of their appearance is the same: their appearance is again needed to understand moduli spaces of theories containing noneffectively gauged sigma models at certain points.

\section{Acknowledgments}

We would like to thank A. Adams, L. Chen, J. Distler, K. Hori, S. Katz, J. McGreevy, and R. Plesser for useful conversations, A. Greenspoon for proofreading, the Aspen Centre for Physics for hospitality while this work was under development, and the UPenn Math-Physics group for the excellent conditions for collaboration it provided during several stages of this work. T.P. was partially supported by NSF grants DMS 0403884 and FRG 0139799 .

\section{Appendix}

\section{Toric stacks}

The paper [2] describes a construction of "toric stacks," which are DeligneMumford stacks over toric varieties.

Toric stacks are described as follows. Begin with a fan describing a toric variety. (The toric stack will naturally live over the toric variety; technically, the toric variety is known as the "moduli space" of the stack, though in this context the term is a holdover from the historical development of stacks and does not refer to any deformation theory.) Enlarge the lattice $N$ to include some finite-group factors, so that if for the original toric variety, $N=\mathbf{Z}^{d}$ for some $d$, then $N$ is now of the form

$$
\mathbf{Z}^{d} \oplus \mathbf{Z}_{q_{1}} \oplus \cdots \oplus \mathbf{Z}_{q_{r}}
$$

For ordinary toric varieties, each edge in the fan describes a map $\mathbf{Z} \rightarrow N$, defined by a generator of that edge. We do the same here - to each edge we 
associate an element of the abelian group $N$ above. The freely generated part of the image corresponds to the coordinates of a generator of the edge, and the finite-group factors will determine the stack structure. The combination of an ordinary fan, $N$, and the maps from edges to $N$ forms what the authors of [2] call a stacky fan.

We can determine $\mathbf{C}^{\times}$charges in a gauged-linear-sigma-model-style presentation as follows. Let $n$ be the number of edges in the fan and $d$ the dimension of the freely generated part of $N$. Define a $(d+r) \times r$ matrix $Q$ to have 0 's in its first $d$ rows, then $q_{i}$ 's on the diagonal in the last $r$ rows, giving a matrix of the form

$$
Q=\left[\begin{array}{cccc}
0 & 0 & \cdots & 0 \\
& & \cdots & \\
0 & 0 & \cdots & 0 \\
q_{1} & \cdots & 0 & 0 \\
0 & q_{2} & \cdots & 0 \\
0 & \cdots & q_{r-1} & 0 \\
0 & 0 & \cdots & q_{r}
\end{array}\right] .
$$

Define a $(d+r) \times n$ matrix $P$ to have, in each column, the image in $N$ of the corresponding edge. Then, the gauged-linear-sigma-model charges are defined by the kernel of the $(d+r) \times(n+r)$ matrix $[P Q]$, a result in close analogy with the way gauged-linear-sigma-model charges are computed from ordinary fans for ordinary toric varieties. Technically there can sometimes be additional finite-group actions beyond the $\mathbf{C}^{\times}$'s; we shall return to this matter after describing a few basic examples.

Our first example is also example 2.1 in [2]. The underlying toric variety is $\mathbf{P}^{1}$, with its trivial fan, and take $N=\mathbf{Z} \oplus \mathbf{Z}_{2}$. Since the toric variety is one-dimensional, $d=1$, and since there is only one finite-group factor $r=1$, and in fact the matrix $Q$ is given by

$$
\left[\begin{array}{l}
0 \\
2
\end{array}\right]
$$

To the first edge, we associate $(2,1) \in N$, and to the second edge we associate $(-3,0) \in N$. Thus, the matrix $P$ is given by

$$
\left[\begin{array}{cc}
2 & -3 \\
1 & 0
\end{array}\right]
$$

The gauged-linear-sigma-model charges are computed as the solutions $a, b$, $c$ of the equation

$$
\left[\begin{array}{ccc}
2 & -3 & 0 \\
1 & 0 & 2
\end{array}\right]\left[\begin{array}{l}
a \\
b \\
c
\end{array}\right]=0
$$


The possible solutions are generated by

$$
\left[\begin{array}{lll}
a & b & c
\end{array}\right]=\left[\begin{array}{lll}
6 & 4 & -3
\end{array}\right]
$$

We discard the value of $c$, and then the resulting stack can be realized by a gauged linear sigma model acting on two chiral superfields, with a single $U(1)$, under which the chiral superfields have charges 6,4 . Truncating $c$ may seem somewhat artificial, but has a natural mathematical interpretation, which we shall return to later.

Suppose we modify the example above slightly, using the same underlying toric fan and the same $N$, but we modify the maps from the edges into $N$ so that they produce no torsion, i.e., to the edges we associate $(2,0)$ and $(-3,0)$. Now when we compute the gauged linear sigma model charges as the kernel of

$$
\left[\begin{array}{ccc}
2 & -3 & 0 \\
0 & 0 & 2
\end{array}\right]
$$

we find that the charges are 3,2 . Adding the torsion forces us to use nonminimal charges.

Technically, the example above describes more than just the weighted projective space $W \mathbf{P}_{3,2}$; it is actually the stack $W \mathbf{P}_{3,2} \times B \mathbf{Z}_{2}$. More generally, putting all 0 's in a row of the $P$ matrix will result in a $B \mathbf{Z}_{r}$ factor.

Let us take a moment to understand the details of the construction in [2] a little better, which will allow us to see the origin of these finite-group factors. The paper [2] defines a group $D G(\beta)$ to be $\left(\mathbf{Z}^{n+r}\right)^{*}$ modulo the image of the matrix $[P Q]^{\mathrm{T}}$. The same reference also defines a map $\beta^{\vee}$, which turns out to be given by the composition of the inclusion

$$
\left(\mathbf{Z}^{n}\right)^{*} \hookrightarrow\left(\mathbf{Z}^{n+r}\right)^{*}
$$

and the projection

$$
\left(\mathbf{Z}^{n+r}\right)^{*} \longrightarrow D G(\beta)
$$

This projection can be accomplished by contracting the elements of $\left(\mathbf{Z}^{n+r}\right)^{\vee}$ with elements of the kernel of $[P Q]$, the same kernel that defines the gauged linear sigma model charges. (The map $\beta$ is the assignment of an element of $N$ to each edge of the fan, thus, a map $\mathbf{Z}^{n} \rightarrow N$.) To compute finitegroup factors and their actions, we need to compute $D G(\beta)$ and $\beta^{\vee}$ in each example. The group one quotients by $G=\operatorname{Hom}\left(D G(\beta), \mathbf{C}^{\times}\right)$. If $D G(\beta)=$ $\mathbf{Z}^{k}$ for some $k$, then $G=\left(\mathbf{C}^{\times}\right)^{k}$, and we have an ordinary gauged linear sigma model. If $D G(\beta)$ contains finite-group factors, then we must quotient by more than merely $\mathbf{C}^{\times}$'s to obtain the toric stack. Furthermore, the action of $G$ on the homogeneous coordinates is defined by the map $\operatorname{Hom}\left(\beta^{\vee}, \mathbf{C}^{\times}\right)$. 
In the first example we discussed above, $D G(\beta)=\mathbf{Z}$. The map $\beta^{\vee}$ is the composition of the inclusion

$$
\left(\mathbf{Z}^{2}\right)^{*} \longrightarrow\left(\mathbf{Z}^{3}\right)^{*}:\left[\begin{array}{l}
a \\
b
\end{array}\right] \longmapsto\left[\begin{array}{l}
a \\
b \\
0
\end{array}\right]
$$

and the projection

$$
\left(\mathbf{Z}^{3}\right)^{*} \longrightarrow \mathbf{Z}:\left[\begin{array}{l}
a \\
b \\
c
\end{array}\right] \longmapsto[64-3]\left[\begin{array}{l}
a \\
b \\
c
\end{array}\right]=6 a+4 b-3 c
$$

More simply, the map $\beta^{\vee}$ is the map

$$
\left(\mathbf{Z}^{2}\right)^{\vee} \longrightarrow \mathbf{Z}:\left[\begin{array}{l}
a \\
b
\end{array}\right] \longmapsto 6 a+4 b
$$

Since $D G(\beta)=\mathbf{Z}, G=\mathbf{C}^{\times}$, and the action of $G$ on the homogeneous coordinates is given by $\operatorname{Hom}\left(\beta^{\vee}, \mathbf{C}^{\times}\right)$or more simply,

$$
(x, y) \longmapsto\left(\lambda^{6} x, \lambda^{4} y\right)
$$

More generally, it is clear from the form of the first inclusion map that the $\mathbf{C}^{\times}$charges of the homogeneous coordinates will be obtained by truncating the kernel vectors.

In the second example we discussed above, $D G(\beta)=\mathbf{Z} \oplus \mathbf{Z}_{2}$. The map $\beta^{\vee}$ is the composition of the inclusion above, plus the projection

$$
\left(\mathbf{Z}^{3}\right)^{*} \longrightarrow \mathbf{Z} \oplus \mathbf{Z}_{2}:\left[\begin{array}{l}
a \\
b \\
c
\end{array}\right] \longmapsto[320]\left[\begin{array}{l}
a \\
b \\
c
\end{array}\right]=3 a+2 b
$$

In other words, the map $\beta^{\vee}$ is the map

$$
\left(\mathbf{Z}^{2}\right)^{\vee} \longrightarrow \mathbf{Z} \oplus \mathbf{Z}_{2}:\left[\begin{array}{l}
a \\
b
\end{array}\right] \longmapsto(3 a+2 b) \oplus 0
$$

Here, $G=\mathbf{C}^{\times} \oplus \mathbf{Z}_{2}$, and dualizing $\beta^{\vee}$, we find that the action of $\mathbf{C}^{\times}$on the homogeneous coordinates is given by

$$
(x, y) \longmapsto\left(\lambda^{3} x, \lambda^{2} y\right)
$$

and the $\mathbf{Z}_{2}$ acts trivially. Thus, here the toric stack is $W \mathbf{P}_{2,3} \times B \mathbf{Z}_{2}$.

We can also realize the gerbe of Section 3.1 in this language. Recall that was a $\mathbf{Z}_{k}$ gerbe over $\mathbf{P}^{N-1}$, which generated all of the $\mathbf{Z}_{k}$ gerbes on $\mathbf{P}^{N-1}$. 
Since it is a $\mathbf{Z}_{k}$ gerbe, the matrix $Q$ is an $N$-element column vector of the form

$$
Q=\left[\begin{array}{c}
0 \\
0 \\
\cdots \\
0 \\
k
\end{array}\right]
$$

and if we take the matrix $P$ to be given by

$$
P=\left[\begin{array}{ccccc}
1 & 0 & \cdots & 0 & -1 \\
0 & 1 & \cdots & 0 & -1 \\
& & \cdots & & \\
0 & 0 & \cdots & 1 & -1 \\
0 & 0 & \cdots & 0 & 1
\end{array}\right]
$$

then column vectors that solve the equation

$$
[P Q]\left[\begin{array}{c}
a_{1} \\
\cdots \\
a_{N} \\
b
\end{array}\right]=0
$$

are generated by

$$
\left[\begin{array}{c}
a_{1} \\
a_{2} \\
\cdots \\
a_{N} \\
b
\end{array}\right]=\left[\begin{array}{c}
k \\
k \\
\cdots \\
k \\
-1
\end{array}\right]
$$

from which we see the gauged-linear-sigma-model-type description in terms of $N$ chiral superfields each of charge $k$. It is straightforward to check that $D G(\beta)=\mathbf{Z}$, so there are no extra finite-group factors here.

We do not need to add any finite groups to the $N$ lattice in order to obtain a stack that is not a space; we can get stacks from the data defining an ordinary toric variety, if it is singular. For a smooth toric variety, if we reinterpret the same data defining the variety as a stack, then the resulting stack will be the same as the original toric variety. However, if we take a singular toric variety, such as the quotient space $\mathbf{C}^{2} / \mathbf{Z}_{2}$, and interpret the fan data as a toric stack, then the resulting stack is not the space $\mathbf{C}^{2} / \mathbf{Z}_{2}$, but rather is the stack $\left[\mathbf{C}^{2} / \mathbf{Z}_{2}\right]$. Similarly, weighted projective stacks are described as toric stacks using the same fan and $N$ lattice as for the corresponding toric varieties, but merely reinterpreted as stacks.

This is how our conjectured extension of Batyrev's mirror proposal to stacks reproduces mirror symmetry for spaces. Interpreted as stacks, toric 
varieties such as the quotient space $\mathbf{P}^{2} / \mathbf{Z}_{3}$ are replaced by toric stacks $\left[\mathbf{P}^{2} / \mathbf{Z}_{3}\right]$, reproducing orbifold mirrors.

\section{References}

[1] T. Pantev and E. Sharpe, String compactifications on Calabi-Yau stacks, hep-th/0502044.

[2] L. Borisov, L. Chen and G. Smith, The orbifold Chow ring of toric Deligne-Mumford stacks, math.AG/0309229.

[3] D. Morrison and R. Plesser, Summing the instantons: quantum cohomology and mirror symmetry in toric varieties, Nucl. Phys. B440 (1995) 279-354; hep-th/9412236.

[4] T. Pantev and E. Sharpe, Notes on gauging noneffective group actions, hep-th/0502027.

[5] E. Witten, Instantons, the quark model, and the $1 / N$ expansion, Nucl. Phys. B149 (1979) 285-320.

[6] K. Hori and C. Vafa, Mirror symmetry, hep-th/0002222.

[7] E. Witten, Phases of $\mathcal{N}=2$ theories in two dimensions, Nucl. Phys. B403 (1993) 159-222; hep-th/9301042.

[8] R. Plesser, private communication.

[9] D. Abramovich, T. Graber and A. Vistoli, Algebraic orbifold quantum products, math.AG/0112004.

[10] D. Morrison and R. Plesser, Towards mirror symmetry as duality for two-dimensional abelian gauge theories, Nucl. Phys. Proc. Suppl. 46 (1996) 177-186; hep-th/9508107.

[11] C. Vafa, Topological Landau-Ginzburg models, Mod. Phys. Lett. A6 (1991) 337-346.

[12] M. Aganagic and C. Vafa, Mirror symmetry and supermanifolds, hep-th/0403192.

[13] V. Batyrev, Dual polyhedra and mirror symmetry for Calabi-Yau hypersurfaces in toric varieties, alg-geom/9310003.

[14] P. Candelas, X. de la Ossa and S. Katz, Mirror symmetry for CalabiYau hypersurfaces in weighted $\mathbf{P}^{4}$ and extensions of Landau-Ginzburg theory, Nucl. Phys. B450 (1995) 267-292; hep-th/9412117. 
